\title{
On Differential-Algebraic Equations in Infinite Dimensions
}

\author{
Sascha Trostorff \& Marcus Waurick
}

\begin{abstract}
We consider a class of differential-algebraic equations (DAEs) with index zero in an infinite dimensional Hilbert space. We define a space of consistent initial values, which lead to classical continuously differential solutions for the associated DAE. Moreover, we show that for arbitrary initial values we obtain mild solutions for the associated problem. We discuss the asymptotic behaviour of solutions for both problems. In particular, we provide a characterisation for exponential stability and exponential dichotomies in terms of the spectrum of the associated operator pencil.
\end{abstract}

Keywords and phrases: Differential-algebraic equations, consistent initial values, strong and mild solutions, exponential stability, exponential dichotomy

Mathematics subject classification 2010: 34A09, 34A12, 34D20, 34D09, 46N20, 47N20

\section{Introduction}

Let $H$ be a Hilbert space and let $M_{0}, M_{1}$ be bounded linear operators in $H$. In this article we are concerned with the implicit initial value problem of finding $u: \mathbb{R}_{\geq 0} \rightarrow H$ such that

$$
\left\{\begin{array}{l}
M_{0} u^{\prime}(t)+M_{1} u(t)=0, \quad t>0, \\
u(0+)=u_{0}
\end{array}\right.
$$

for some given initial datum $u_{0} \in H$, where (図) has to be interpreted in a particular sense. This problem is commonly known as a "differential-algebraic equation" and has attracted a lot of interest in recent years. Most prominently, for finite-dimensional $H$, differential-algebraic equations have applications to control and electrical circuit theory, see e.g. 2]. For so-called regular systems and a finite-dimensional state space $H$, the discussion of (荬 can be simplified by applying a generalised Jordan normal form, see e.g. 1, 2, 5. A similar strategy can be used for particular infinite-dimensional cases, see [13. We are unaware of any other treatment of differential-algebraic equations in infinite spatial dimensions. Thus, for the convenience of the reader, we present this work as much as self-contained as possible.

Our main motivation for discussing the infinite-dimensional case comes from the viewpoint of so-called evolutionary equations, a certain class of partial differential-algebraic 
equations introduced in [10]. The focus of [10] was to derive a particular Hilbert space setting such that a large class of linear equations in mathematical physics can be dealt with in a unified framework. A particular case is the equation

$$
\left(M_{0} u\right)^{\prime}+M_{1} u+A u=f
$$

for some (unbounded, skew-selfadjoint) operator $A$ in $H$. In order to cover a large class of problems and to allow for $M_{0}=0$, as well, the basic setting in [10] uses homogeneous initial conditions. This, on the other hand, lead to the question of what possible initial values can be assumed for (11) in order to have a solution $u$.

In the habilitation thesis [18 this question has been addressed. In this article we shall carry out a more detailed analysis for the case of when $A=0$. For this reason, we shall furthermore focus on the case of regular systems with index 0 . Moreover, we will assume that $R\left(M_{0}\right) \subseteq H$ is closed.

Under the assumptions mentioned, we will derive a space of admissible initial values $u_{0}$

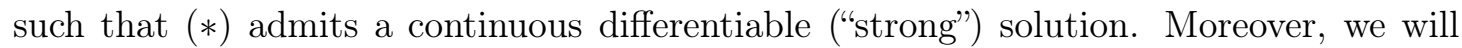
discuss the regularity of solutions for (因), if $u_{0}$ is not admissible. It turns out that the correct notion of a solution can be phrased as a "mild" solution, that is, $u$ satisfies (因) only in an once integrated sense. Having obtained well-posedness of (因), we will address the asymptotic behaviour of both strong and mild solutions. We will formulate a spectral characterisation of both mild and strong exponential dichotomy as well as mild and strong exponential stability and will recover the well-known finite-dimensional stability results, see e.g. [3, 2, 15]. We emphasise that in our approach we do not use the generalised Jordan normal form or Weierstrass normal form, simply because these strategies are not applicable under the assumptions stated.

A more thorough analysis of the case of non zero index has been initiated in [19] and will be addressed in future work.

We shall comment on the organisation of this text. In the next section, we gather some material on evolutionary equations and establish the time-derivative as a suitable continuously invertible operator in a weighted vector-valued $L_{2}$-space. In Section 3 we introduce our central object of study and define regular linear operator pencils (of index 0 ). We also provide a characterisation of regularity given the closedness of the range of $M_{0}$. The characterisation of admissible initial values $u_{0}$ such that (荬) has a continuous differentiable solution will be given in Section 4 Mild solutions, that is, where (因) holds in an integrated sense, only, will be considered in Section 5. It turns out that the problem of finding continuously differentiable solutions to (荬 can be written as an abstract Cauchy problem leading to a norm-continuous semigroup as a fundamental solution. The spectrum of the generator of this semigroup is shown to coincide with the spectrum of the operator pencil in Section 6. In Section 7 we address the asymptotics (exponential dichotomy) of both the derived Cauchy problem as well as for mild solutions. Note that the difficult part is to define an appropriate notion for the case of mild solutions as they are a priori only locally integrable. 


\section{Preliminaries}

Following [10, 4, 9], we introduce the temporal derivative as a normal operator in an exponentially weighted $L_{2}$-space. One can view the exponential weight as an $L_{2}$-variant of the well-known Morgenstern norm that is used for proving the Picard-Lindelöf theorem for arbitrary large Lipschitz constant, see [7] or [11, Section 4] for a more recent reference.

Definition. Let $\rho \in \mathbb{R}$. We define the Hilbert space $L_{2, \rho}(\mathbb{R} ; H)$ of (equivalence classes of) $\mathrm{H}$-valued functions as follows

$$
L_{2, \rho}(\mathbb{R} ; H):=\left\{f: \mathbb{R} \rightarrow H ; f \text { measurable, } \int_{\mathbb{R}}|f(t)|_{H}^{2} \exp (-2 \rho t) \mathrm{d} t<\infty\right\},
$$

equipped with the usual inner product

$$
\langle f, g\rangle_{\rho}:=\int_{\mathbb{R}}\langle f(t), g(t)\rangle_{H} \exp (-2 \rho t) \mathrm{d} t .
$$

Moreover, we define the operator $\partial_{0, \rho}: H_{\rho}^{1}(\mathbb{R} ; H) \subseteq L_{2, \rho}(\mathbb{R} ; H) \rightarrow L_{2, \rho}(\mathbb{R} ; H)$ as the closure of

$$
\begin{aligned}
C_{c}^{\infty}(\mathbb{R} ; H) \subseteq L_{2, \rho}(\mathbb{R} ; H) & \rightarrow L_{2, \rho}(\mathbb{R} ; H), \\
\phi & \mapsto \phi^{\prime}
\end{aligned}
$$

where $C_{c}^{\infty}(\mathbb{R} ; H)$ denotes the space of arbitrarily differentiable functions from $\mathbb{R}$ to $H$ with compact support.

Note that every continuous function $f: \mathbb{R} \rightarrow H$ with support bounded below that satisfies the exponential growth condition $|f(t)|_{H} \leq M e^{\omega t}$ for some $\omega \in \mathbb{R}, M \geq 0$ and all $t \in \mathbb{R}$ belongs to $L_{2, \rho}(\mathbb{R} ; H)$ for all $\rho>\omega$. We shall need this observation later on. Some more remarks are in order.

Remark 2.1. (a) For $\rho=0$ the space $L_{2,0}(\mathbb{R} ; H)$ is nothing but the standard $L_{2}$-space of Bochner-measurable functions with values in $H$. Moreover, $\partial_{0,0}$ is the usual weak derivative on $L_{2}$.

(b) The domain $H_{\rho}^{1}(\mathbb{R} ; H)$ is an exponentially weighted variant of the Sobolev space $H^{1}(\mathbb{R} ; H)$ and becomes a Hilbert space with respect to the graph inner product of $\partial_{0, \rho}$, that is,

$$
\langle f, g\rangle_{\rho, 1}:=\langle f, g\rangle_{\rho}+\left\langle\partial_{0, \rho} f, \partial_{0, \rho} g\right\rangle_{\rho} .
$$

It turns out that the operator $\partial_{0, \rho}$ is a normal operator, whose spectral representation is given in terms of the so-called Fourier-Laplace transform, which is defined as follows.

Proposition 2.2 (4, Corollary 2.5]). Let $\rho \in \mathbb{R}$. Then the operator

$$
\left.\mathcal{L}_{\rho}\right|_{C_{c}^{\infty}(\mathbb{R} ; H)}: C_{c}^{\infty}(\mathbb{R} ; H) \subseteq L_{2, \rho}(\mathbb{R} ; H) \rightarrow L_{2,0}(\mathbb{R} ; H)
$$




$$
\phi \mapsto\left(t \mapsto \frac{1}{\sqrt{2 \pi}} \int_{\mathbb{R}} \exp (-(\mathrm{i} t+\rho) s) \phi(s) \mathrm{d} s\right)
$$

has a unitary extension to $L_{2, \rho}(\mathbb{R} ; H)$, which will be denoted by $\mathcal{L}_{\rho}$. Moreover,

$$
\partial_{0, \rho}=\mathcal{L}_{\rho}^{*}(\mathrm{i} \mathrm{m}+\rho) \mathcal{L}_{\rho},
$$

where $\mathrm{m}: D(\mathrm{~m}) \subseteq L_{2,0}(\mathbb{R} ; H) \rightarrow L_{2,0}(\mathbb{R} ; H)$ is given by

$$
\mathrm{m} f:=(t \mapsto t f(t))
$$

for $f \in D(\mathrm{~m}):=\left\{g \in L_{2,0}(\mathbb{R} ; H) ;(t \mapsto t g(t)) \in L_{2,0}(\mathbb{R} ; H)\right\}$.

Remark 2.3. In the case $\rho=0, \mathcal{L}_{0}$ coincides with the usual Fourier transform on $L_{2}$, which is unitary by Plancherel's Theorem. For general $\rho$ we obtain $\mathcal{L}_{\rho}=\mathcal{L}_{0} \exp (-\rho \mathrm{m})$, where the operator $\exp (-\rho \mathrm{m})$ given by $(\exp (-\rho \mathrm{m}) f)(t):=\exp (-\rho t) f(t)$ is obviously unitary from $L_{2, \rho}$ to $L_{2,0}$. Hence, the unitarity of $\mathcal{L}_{\rho}$ follows.

Using the spectral representation for $\partial_{0, \rho}$, we can easily show the following properties.

Corollary 2.4. Let $\rho \in \mathbb{R}$.

(a) The spectrum of $\partial_{0, \rho}$ is given by $\sigma\left(\partial_{0, \rho}\right)=\{z \in \mathbb{C} ; \operatorname{Re} z=\rho\}$.

(b) The adjoint of $\partial_{0, \rho}$ is given by $\partial_{0, \rho}^{*}=-\partial_{0, \rho}+2 \rho$.

(c) If $\rho \neq 0$, then $\partial_{0, \rho}$ is continuously invertible with

$$
\left(\partial_{0, \rho}^{-1} f\right)(t)=\left\{\begin{array}{ll}
\int_{-\infty}^{t} f(s) \mathrm{d} s & \text { if } \rho>0, \\
-\int_{t}^{\infty} f(s) \mathrm{d} s & \text { if } \rho<0,
\end{array} \quad\left(t \in \mathbb{R}, f \in L_{2, \rho}(\mathbb{R} ; H)\right) .\right.
$$

We conclude the section with the following variant of the Sobolev-embedding theorem.

Proposition 2.5 ([4, Lemma 5.2]). Let $\rho \in \mathbb{R}$ and define

$$
C_{\rho, 0}(\mathbb{R} ; H):=\left\{f: \mathbb{R} \rightarrow H ; f \text { continuous, } \lim _{t \rightarrow \pm \infty} f(t) \exp (-\rho t)=0\right\},
$$

equipped with the norm

$$
|f|_{\rho, \infty}:=\sup \left\{|f(t)|_{H} \exp (-\rho t) ; t \in \mathbb{R}\right\}
$$

Then $H_{\rho}^{1}(\mathbb{R} ; H) \hookrightarrow C_{\rho, 0}(\mathbb{R} ; H)$.

In the next section, we recall the notion of regularity for differential-algebraic equations, see e.g. [1, 6]. Note that we shall deviate slightly from the usual notion of regularity in as much as we restrict our consideration to index 0, only. For differential-algebraic equations with nontrivial index, we refer to the study initiated in [19]. 


\section{Regular linear operator pencils}

Throughout this section, let $M_{0}, M_{1} \in L(H)$.

Definition. We consider the function

$$
\begin{aligned}
\mathcal{M}: \mathbb{C} & \rightarrow L(H) \\
z & \mapsto z M_{0}+M_{1}
\end{aligned}
$$

and call $\mathcal{M}$ the linear operator pencil associated with $\left(M_{0}, M_{1}\right)$. We define the spectrum of $\mathcal{M}$ by

$$
\sigma(\mathcal{M}):=\left\{z \in \mathbb{C} ; 0 \in \sigma\left(z M_{0}+M_{1}\right)\right\}
$$

and the resolvent set of $\mathcal{M}$ by

$$
\varrho(\mathcal{M}):=\mathbb{C} \backslash \sigma(\mathcal{M}) .
$$

We call $\mathcal{M}$ regular (of index 0 ), if there exists $\nu \in \mathbb{R}$ such that:

(a) $\mathbb{C}_{\operatorname{Re}>\nu} \subseteq \varrho(\mathcal{M})$ and

(b) $\mathbb{C}_{\operatorname{Re}>\nu} \ni z \mapsto\left(z M_{0}+M_{1}\right)^{-1} \in L(H)$ is bounded.

Moreover, we set

$$
s_{0}(\mathcal{M}):=\inf \{\nu \in \mathbb{R} ;(a) \text { and }(b) \text { are satisfied }\} .
$$

Example 3.1. A standard example for a regular linear pencil is the following. Assume that $M_{0}$ is selfadjoint. Then we can decompose the underlying space $H$ as $N\left(M_{0}\right) \oplus \overline{R\left(M_{0}\right)}$ by the projection theorem. Assume now that $M_{0}$ is strictly accretive on $R\left(M_{0}\right)$ and $M_{1}$ is strictly accretive on $N\left(M_{0}\right)$, i.e. there is $c>0$ such that

$$
\begin{aligned}
\left\langle M_{0} x, x\right\rangle_{H} & \geq c|x|_{H}^{2} \\
\left\langle M_{1} y, y\right\rangle_{H} & \geq c|y|_{H}^{2}
\end{aligned}
$$

for each $x \in R\left(M_{0}\right)$ and $y \in N\left(M_{0}\right)$. Then, the linear pencil associated with $\left(M_{0}, M_{1}\right)$ is regular. Note that in the context of partial differential equations the mentioned positive definiteness conditions happen to be satisfied in many applications, see [10, Section 3.3.3].

We note that in the latter example, the strict accretivity of $M_{0}$ on $R\left(M_{0}\right)$ in particular implies that $R\left(M_{0}\right)$ is closed. In this case, we can characterise the regularity of the pencil by the invertibility of a particular operator. For the next statement, we introduce for a closed subspace $S \subseteq H$ the canonical embedding

$$
\iota_{S}: S \hookrightarrow H, s \mapsto s .
$$

It is easy to see that $\iota_{S}^{*}: H \rightarrow S$ is surjective and acts as the orthogonal projection onto $S$, see also [12, Lemma 3.2]. 
Proposition 3.2. Assume that $R\left(M_{0}\right)$ is closed and denote by $\mathcal{M}$ the linear pencil associated with $\left(M_{0}, M_{1}\right)$. Then the following statements are equivalent:

(i) $\mathcal{M}$ is regular,

(ii) $\iota_{R\left(M_{0}\right)^{\perp}}^{*} M_{1} \iota_{N\left(M_{0}\right)}: N\left(M_{0}\right) \rightarrow R\left(M_{0}\right)^{\perp}$ is continuously invertible.

In the latter case, $\sigma(\mathcal{M})=\sigma\left(-\widetilde{M}_{0}^{-1} \widetilde{M}_{1}\right)$ is compact and for each $z \in \mathbb{C}$ we have that

$$
\mathcal{M}(z)=U_{1}^{*} V_{1}\left(\begin{array}{cc}
\left(z \widetilde{M}_{0}+\widetilde{M}_{1}\right) & 0 \\
0 & \iota_{R\left(M_{0}\right)^{\perp}}^{*} M_{1} \iota_{N\left(M_{0}\right)}
\end{array}\right) V_{0} U_{0},
$$

where

$$
\begin{aligned}
& U_{0}:=\left(\begin{array}{c}
\iota_{N\left(M_{0}\right)^{\perp}}^{*} \\
\iota_{N\left(M_{0}\right)}^{*}
\end{array}\right): H \rightarrow N\left(M_{0}\right)^{\perp} \oplus N\left(M_{0}\right), \\
& U_{1}:=\left(\begin{array}{c}
\iota_{R\left(M_{0}\right)}^{*} \\
\iota_{R\left(M_{0}\right)^{\perp}}^{*}
\end{array}\right): H \rightarrow R\left(M_{0}\right) \oplus R\left(M_{0}\right)^{\perp},
\end{aligned}
$$

and

$$
\begin{aligned}
& V_{0}:=\left(\begin{array}{cc}
1 & 0 \\
\left(\iota_{R\left(M_{0}\right)^{\perp}}^{*} M_{1} \iota_{N\left(M_{0}\right)}\right)^{-1}\left(\iota_{R\left(M_{0}\right)^{\perp}}^{*} M_{1} \iota_{N\left(M_{0}\right)^{\perp}}\right) & 1
\end{array}\right) \in L\left(N\left(M_{0}\right)^{\perp} \oplus N\left(M_{0}\right)\right), \\
& V_{1}:=\left(\begin{array}{cc}
1 & \left(\iota_{R\left(M_{0}\right)}^{*} M_{1} \iota_{N\left(M_{0}\right)}\right)\left(\iota_{R\left(M_{0}\right)^{\perp}}^{*} M_{1} \iota_{N\left(M_{0}\right)}\right)^{-1} \\
0 & 1
\end{array}\right) \in L\left(R\left(M_{0}\right) \oplus R\left(M_{0}\right)^{\perp}\right),
\end{aligned}
$$

as well as

$$
\begin{aligned}
\widetilde{M}_{0}= & \iota_{R\left(M_{0}\right)}^{*} M_{0} \iota_{N\left(M_{0}\right)^{\perp}} \\
\widetilde{M}_{1}= & \iota_{R\left(M_{0}\right)}^{*} M_{1} \iota_{N\left(M_{0}\right)^{\perp}} \\
& -\left(\iota_{R\left(M_{0}\right)}^{*} M_{1} \iota_{N\left(M_{0}\right)}\right)\left(\iota_{R\left(M_{0}\right)^{\perp}}^{*} M_{1} \iota_{N\left(M_{0}\right)}\right)^{-1}\left(\iota_{R\left(M_{0}\right)^{\perp}}^{*} M_{1} \iota_{N\left(M_{0}\right)^{\perp}}\right) .
\end{aligned}
$$

Proof. (i) $\Rightarrow$ (ii): Assume that $\mathcal{M}$ is regular. By the closed graph theorem, it suffices to prove that the operator $\iota_{R\left(M_{0}\right)^{\perp}}^{*} M_{1} \iota_{N\left(M_{0}\right)}$ is bijective. For showing that it is onto, let $f \in R\left(M_{0}\right)^{\perp}$. For $n \in \mathbb{N}$ sufficiently large we define

$$
u_{n}:=\left(n M_{0}+M_{1}\right)^{-1} \iota_{R\left(M_{0}\right)^{\perp}} f .
$$

Then, $\left(u_{n}\right)_{n}$ is bounded and thus, by passing to a suitable subsequence (not relabelled), we can assume that it is weakly convergent. We denote its weak limit by $u$. Then

$$
M_{0} u=\underset{n \rightarrow \infty}{\mathrm{w}-\lim _{0}} M_{0} u_{n}=\underset{n \rightarrow \infty}{\mathrm{w}-\lim _{n}} \frac{1}{n}\left(n M_{0}+M_{1}\right) u_{n}=\underset{n \rightarrow \infty}{\mathrm{w}-\lim _{n}} \frac{1}{n} \iota_{R\left(M_{0}\right)^{\perp}} f=0,
$$


i.e., $u \in N\left(M_{0}\right)$. Moreover, since

$$
\iota_{R\left(M_{0}\right)^{\perp}}^{*} M_{1} u_{n}=\iota_{R\left(M_{0}\right)^{\perp}}^{*}\left(n M_{0}+M_{1}\right) u_{n}=f
$$

for each $n \in \mathbb{N}$, we infer that

$$
\iota_{R\left(M_{0}\right)^{\perp}}^{*} M_{1} \iota_{N\left(M_{0}\right)} u=f,
$$

which proves that $\iota_{R\left(M_{0}\right)^{\perp}}^{*} M_{1} \iota_{N\left(M_{0}\right)}$ is onto. To prove, that it is also one-to-one, let $u \in N\left(M_{0}\right)$ with $\iota_{R\left(M_{0}\right)^{\perp}}^{*} M_{1} \iota_{N\left(M_{0}\right)} u=0$. Consequently, $M_{1} \iota_{N\left(M_{0}\right)} u \in R\left(M_{0}\right)$ and hence, there is $v \in H$ such that $M_{1} \iota_{N\left(M_{0}\right)} u=M_{0} v$. Since $\left(n M_{0}+M_{1}\right) \iota_{N\left(M_{0}\right)} u=M_{1} \iota_{N\left(M_{0}\right)} u=$ $M_{0} v$ for each $n \in \mathbb{N}$, we derive that

$$
\iota_{N\left(M_{0}\right)} u=\left(n M_{0}+M_{1}\right)^{-1} M_{0} v=\frac{1}{n} v-\frac{1}{n}\left(n M_{0}+M_{1}\right)^{-1} M_{1} v
$$

for sufficiently large $n \in \mathbb{N}$. As the right-hand side tends to 0 as $n$ tends to infinity, we infer $\iota_{N\left(M_{0}\right)} u=0$ and thus, $u=0$.

(ii) $\Rightarrow$ (i): For $z \in \mathbb{C}$ we have that

$$
\begin{aligned}
U_{1} \mathcal{M}(z) U_{0}^{*} & =\left(\begin{array}{cc}
\iota_{R\left(M_{0}\right)}^{*}\left(z M_{0}+M_{1}\right) \iota_{N\left(M_{0}\right)^{\perp}} & \iota_{R\left(M_{0}\right)}^{*}\left(z M_{0}+M_{1}\right) \iota_{N\left(M_{0}\right)} \\
\iota_{R\left(M_{0}\right)^{\perp}}^{*}\left(z M_{0}+M_{1}\right) \iota_{N\left(M_{0}\right)^{\perp}} & \iota_{R\left(M_{0}\right)^{\perp}}^{*}\left(z M_{0}+M_{1}\right) \iota_{N\left(M_{0}\right)}
\end{array}\right) \\
& =\left(\begin{array}{cc}
\iota_{R\left(M_{0}\right)}^{*}\left(z M_{0}+M_{1}\right) \iota_{N\left(M_{0}\right)^{\perp}} & \iota_{R\left(M_{0}\right)^{*}}^{*} M_{1} \iota_{N\left(M_{0}\right)} \\
\iota_{R\left(M_{0}\right)^{\perp}}^{*} M_{1} \iota_{N\left(M_{0}\right)^{\perp}} & \iota_{R\left(M_{0}\right)^{\perp}}^{*} M_{1} \iota_{N\left(M_{0}\right)}
\end{array}\right) \\
& =V_{1}\left(\begin{array}{cc}
\left(z \widetilde{M}_{0}+\widetilde{M}_{1}\right) & 0 \\
0 & \iota_{R\left(M_{0}\right)^{\perp}}^{*} M_{1} \iota_{N\left(M_{0}\right)}
\end{array}\right) V_{0}
\end{aligned}
$$

with $U_{0}, U_{1}, V_{0}, V_{1}, \widetilde{M}_{0}$ and $\widetilde{M}_{1}$ as above. Since $U_{0}, U_{1}$ are unitary and $V_{0}, V_{1}$ are continuously invertible, we obtain using (ii) that $\mathcal{M}(z)$ is invertible, if and only if $\left(z \widetilde{M}_{0}+\widetilde{M}_{1}\right)$ is boundedly invertible. Moreover, there is a constant $C \geq 0$ such that for each $z \in \varrho(\mathcal{M})$

$$
\left\|\mathcal{M}(z)^{-1}\right\| \leq C\left\|\left(z \widetilde{M}_{0}+\widetilde{M}_{1}\right)^{-1}\right\| .
$$

Since $\widetilde{M}_{0}$ is bijective, it is boundedly invertible by the closed graph theorem, and hence,

$$
z \mapsto\left(z \widetilde{M}_{0}+\widetilde{M}_{1}\right)^{-1}=\left(z+\widetilde{M}_{0}^{-1} \widetilde{M}_{1}\right)^{-1} \widetilde{M}_{0}^{-1}
$$

is a well-defined bounded function on $\mathbb{C}_{\operatorname{Re}>\left\|\widetilde{M}_{0}^{-1} \widetilde{M}_{1}\right\|}$ by the Neumann series. In particular, $\sigma(\mathcal{M})=\sigma\left(-\widetilde{M}_{0}^{-1} \widetilde{M}_{1}\right)$ is compact.

Remark 3.3. We note that from the representation of $\mathcal{M}(z)$ in Proposition 3.2 it follows that $\sup _{|z|>R}\left\|\mathcal{M}(z)^{-1}\right\|<\infty$, where $R>0$ is sufficiently large. 


\section{A first initial value problem}

Throughout, let $M_{0}, M_{1} \in L(H)$ such that $R\left(M_{0}\right)$ is closed. Moreover, we assume that the linear operator pencil $\mathcal{M}$ associated with $\left(M_{0}, M_{1}\right)$ is regular. In this section, we are concerned with the following initial value problem

$$
\begin{aligned}
M_{0} u^{\prime}(t)+M_{1} u(t) & =0 \quad(t>0), \\
u(0) & =u_{0},
\end{aligned}
$$

where $u_{0} \in H$ is a given initial value and $u: \mathbb{R}_{\geq 0} \rightarrow H$ is to be determined. We recall that (IVP1) is a differential-algebraic equation. The aim of this section is to find a characterisation of possible initial values $u_{0}$ such that the solution of (IVP1) is continuously differentiable on $\mathbb{R}_{>0}$. We present a necessary condition for $u_{0}$ in the next statement. The main result of this section will be to identify this necessary condition also as sufficient, see Corollary 4.5 below.

Lemma 4.1. If $u: \mathbb{R}_{\geq 0} \rightarrow H$ is continuous, $\left.u\right|_{\mathbb{R}_{>0}}$ is continuously differentiable and if u satisfies (IVP1), then

$$
u_{0} \in \mathrm{IV}:=\left\{x \in H ; M_{1} x \in R\left(M_{0}\right)\right\}=M_{1}^{-1}\left[R\left(M_{0}\right)\right] .
$$

Proof. Since we have

$$
M_{1} u(t)=-M_{0} u^{\prime}(t) \in R\left(M_{0}\right)
$$

for each $t>0$, we infer

$$
M_{1} u_{0}=M_{1} u(0) \in R\left(M_{0}\right)
$$

due to the closedness of $R\left(M_{0}\right)$, i.e. $u_{0} \in \mathrm{IV}$.

Remark 4.2. Note that IV is a closed subspace of $H$, as it is the pre-image of the closed subspace $R\left(M_{0}\right)$ under $M_{1}$.

For the converse of Lemma 4.1, we need a couple of preparations.

Proposition 4.3. The operator

$$
\iota_{R\left(M_{0}\right)}^{*} M_{0} \iota_{\mathrm{IV}}: \mathrm{IV} \rightarrow R\left(M_{0}\right)
$$

is bijective and hence, continuously invertible.

Proof. To show that the operator is one-to-one, let $x \in \mathrm{IV} \cap N\left(M_{0}\right)$. Hence, there is $y \in H$ such that $M_{1} x=M_{0} y$ and consequently,

$$
\left(n M_{0}+M_{1}\right) x=M_{1} x=M_{0} y
$$

for each $n \in \mathbb{N}$. Thus, for $n$ large enough, we get

$$
x=\left(n M_{0}+M_{1}\right)^{-1} M_{0} y=\frac{1}{n} y-\frac{1}{n}\left(n M_{0}+M_{1}\right)^{-1} M_{1} y
$$


and since $\lim \sup _{n \rightarrow \infty}\left\|\left(n M_{0}+M_{1}\right)^{-1}\right\|<\infty$, we infer $x=0$. Thus, $\iota_{R\left(M_{0}\right)}^{*} M_{0} \iota_{\mathrm{IV}}$ is one-to-one. For showing that it is onto, let $w \in R\left(M_{0}\right)$. For $n \in \mathbb{N}$ large enough, we set

$$
u_{n}:=\left(n M_{0}+M_{1}\right)^{-1} n w .
$$

We claim that $u_{n} \in \mathrm{IV}$. Indeed, using that

$$
\begin{aligned}
M_{1}\left(n M_{0}+M_{1}\right)^{-1} M_{0} & =\frac{1}{n} M_{1}\left(1-\left(n M_{0}+M_{1}\right)^{-1} M_{1}\right) \\
& =\frac{1}{n} M_{1}-\frac{1}{n}\left(M_{1}-n M_{0}\left(n M_{0}+M_{1}\right)^{-1} M_{1}\right) \\
& =M_{0}\left(n M_{0}+M_{1}\right)^{-1} M_{1},
\end{aligned}
$$

we infer that

$$
\left(n M_{0}+M_{1}\right)^{-1}\left[R\left(M_{0}\right)\right] \subseteq \mathrm{IV} .
$$

Since $n w \in R\left(M_{0}\right)$, we derive the claim. Moreover, we find $y \in H$ such that $w=M_{0} y$ and we compute for all $n \in \mathbb{N}$

$$
u_{n}=\left(n M_{0}+M_{1}\right)^{-1} n w=\left(n M_{0}+M_{1}\right)^{-1} n M_{0} y=y-\left(n M_{0}+M_{1}\right)^{-1} M_{1} y,
$$

which shows that $\left(u_{n}\right)_{n \in \mathbb{N}}$ is a bounded sequence in IV $\subseteq H$ and hence, by passing to a suitable subsequence, we may assume without loss of generality that $u_{n} \rightarrow u$ for some $u \in \mathrm{IV}$. We obtain

$$
M_{0} \iota_{\mathrm{IV}} u=\underset{n \rightarrow \infty}{\mathrm{w}-\lim _{0}} M_{0} u_{n}=\underset{n \rightarrow \infty}{\mathrm{w}-\lim } \frac{1}{n}\left(n M_{0}+M_{1}\right) u_{n}=w,
$$

which proves the assertion.

Remark 4.4. We shall note here that IV and $N\left(M_{0}\right)^{\perp}$ are isomorphic as Banach spaces. Indeed, $\iota_{R\left(M_{0}\right)}^{*} M_{0} \iota_{N\left(M_{0}\right)^{\perp}}$ is bijective and closed, and hence, a Banach space isomorphism. By Proposition 4.3, $\iota_{R\left(M_{0}\right)}^{*} M_{0} \iota_{\mathrm{IV}}$ is a Banach space isomorphism, as well. So,

$$
\left(\iota_{R\left(M_{0}\right)}^{*} M_{0} \iota_{\mathrm{IV}}\right)^{-1} \iota_{R\left(M_{0}\right)}^{*} M_{0} \iota_{N\left(M_{0}\right)^{\perp}}: N\left(M_{0}\right)^{\perp} \rightarrow \mathrm{IV}
$$

yields the desired isomorphism.

Corollary 4.5. Let $u_{0} \in \mathrm{IV}$. Then there exists a unique solution $u: \mathbb{R}_{\geq 0} \rightarrow H$ of (IVP1), which is given by

$$
u(t)=\exp \left(-t\left(\iota_{R\left(M_{0}\right)}^{*} M_{0} \iota_{\mathrm{IV}}\right)^{-1}\left(\iota_{R\left(M_{0}\right)}^{*} M_{1} \iota_{\mathrm{IV}}\right)\right) u_{0} \quad(t \geq 0) .
$$

Proof. We address uniqueness first: If $u: \mathbb{R}_{\geq 0} \rightarrow H$ is a solution of (IVP1), then $M_{1} u(t)=-M_{0} u^{\prime}(t) \in R\left(M_{0}\right)$. Hence, $u$ attains values in IV. Thus, equivalently,

$$
\iota_{R\left(M_{0}\right)}^{*} M_{1} \iota_{\mathrm{IV}} u(t)=-\iota_{R\left(M_{0}\right)}^{*} M_{0} \iota_{\mathrm{IV}} u^{\prime}(t)
$$


and by Proposition 4.3

$$
u^{\prime}(t)=-\left(\iota_{R\left(M_{0}\right)}^{*} M_{0} \iota_{\mathrm{IV}}\right)^{-1}\left(\iota_{R\left(M_{0}\right)}^{*} M_{1} \iota_{\mathrm{IV}}\right) u(t)
$$

for $t>0$. Hence,

$$
u(t)=\exp \left(-t\left(\iota_{R\left(M_{0}\right)}^{*} M_{0} \iota_{\mathrm{IV}}\right)^{-1}\left(\iota_{R\left(M_{0}\right)}^{*} M_{1} \iota_{\mathrm{IV}}\right)\right) u_{0} \quad(t \geq 0) .
$$

For the existence part consider

$$
\mathbb{R}_{\geq 0} \ni t \mapsto u(t)=\exp \left(-t\left(\iota_{R\left(M_{0}\right)}^{*} M_{0} \iota_{\mathrm{IV}}\right)^{-1}\left(\iota_{R\left(M_{0}\right)}^{*} M_{1} \iota_{\mathrm{IV}}\right)\right) u_{0}
$$

Then clearly $u(0)=u_{0}$ and

$$
u^{\prime}(t)=-\left(\iota_{R\left(M_{0}\right)}^{*} M_{0} \iota_{\mathrm{IV}}\right)^{-1}\left(\iota_{R\left(M_{0}\right)}^{*} M_{1} \iota_{\mathrm{IV}}\right) u(t)
$$

which in turn implies

$$
M_{0} u^{\prime}(t)+M_{1} u(t)=0
$$

for each $t>0$. Hence, $u$ solves (IVP1).

\section{A second initial value problem}

In this section, we aim at providing a different perspective to the initial value problem stated in (IVP1). In the previous section, we have addressed finding solutions $u$ in the "strong sense", that is, we were looking for continuously differentiable solutions. The differentiability, in turn, restricted the class of admissible initial values. Here, we study the solvability of the differential-algebraic equation in a "weak" or "mild" sense. Weakening the solution concepts, we will be able to solve the differential-algebraic equation for all initial data from $H$. For this, we provide a different solution representation of the solution to (IVP1).

Any function defined on $\mathbb{R}_{\geq 0}$ is considered to be a function on $\mathbb{R}$ by extension by 0 . The support of a function $f$ will be denoted by $\operatorname{spt} f$.

Proposition 5.1. Let $u_{0} \in \mathrm{IV}$ and $u: \mathbb{R}_{\geq 0} \rightarrow H$ the solution of (IVP1). Then there is $\rho_{0} \geq 0$ such that $u \in \bigcap_{\rho>\rho_{0}} L_{2, \rho}(\mathbb{R} ; H)$ and

$$
\left(\mathcal{L}_{\rho} u\right)(t)=\frac{1}{\sqrt{2 \pi}}\left((\mathrm{i} t+\rho) M_{0}+M_{1}\right)^{-1} M_{0} u_{0} \quad\left(\rho>\rho_{0}, t \in \mathbb{R}\right) .
$$

Proof. Since by Corollary 4.5

$$
u(t)=\exp \left(-t\left(\iota_{R\left(M_{0}\right)}^{*} M_{0} \iota_{\mathrm{IV}}\right)^{-1}\left(\iota_{R\left(M_{0}\right)}^{*} M_{1} \iota_{\mathrm{IV}}\right)\right) u_{0}
$$


for $t \geq 0$, we infer that $|u(t)| \leq M \mathrm{e}^{\rho_{1} t}$ for some $M \geq 1, \rho_{1} \in \mathbb{R}$ and all $t \geq 0$. Hence, using that spt $u \subseteq \mathbb{R}_{\geq 0}$, we obtain $u \in \bigcap_{\rho>\rho_{1}} L_{2, \rho}(\mathbb{R} ; H)$. We define $\rho_{0}:=\max \left\{0, \rho_{1}, s_{0}(\mathcal{M})\right\}$ and claim that

$$
u-\chi_{\mathbb{R}_{\geq 0}} u_{0} \in \bigcap_{\rho>\rho_{0}} H_{\rho}^{1}(\mathbb{R} ; H) .
$$

Indeed, for $\rho>\rho_{0}$ we have that $u-\chi_{\mathbb{R}_{\geq 0}} u_{0} \in L_{2, \rho}(\mathbb{R} ; H)$ and for $\varphi \in C_{c}^{\infty}(\mathbb{R} ; H)$ we compute using Corollary 2.4

$$
\begin{aligned}
\left\langle u-\chi_{\mathbb{R}_{\geq 0}} u_{0}, \partial_{0, \rho}^{*} \varphi\right\rangle_{\rho} & =\int_{0}^{\infty}\left\langle u(t)-u_{0},-\varphi^{\prime}(t)+2 \rho \varphi(t)\right\rangle_{H} \mathrm{e}^{-2 \rho t} \mathrm{~d} t \\
& =\int_{0}^{\infty}\left\langle u^{\prime}(t), \varphi(t)\right\rangle_{H} \mathrm{e}^{-2 \rho t} \mathrm{~d} t \\
& =\left\langle u^{\prime}, \varphi\right\rangle_{\rho}
\end{aligned}
$$

by integration by parts, and thus $u-\chi_{\mathbb{R}_{\geq 0}} u_{0} \in H_{\rho}^{1}(\mathbb{R} ; H)$ with $\partial_{0, \rho}\left(u-\chi_{\mathbb{R}_{\geq 0}} u_{0}\right)=u^{\prime}$. Hence, we compute with Proposition 2.2

$$
\begin{aligned}
& \left((\mathrm{i} t+\rho) M_{0}+M_{1}\right)\left(\mathcal{L}_{\rho} u\right)(t) \\
& =(\mathrm{i} t+\rho) M_{0}\left(\mathcal{L}_{\rho}\left(u-\chi_{\mathbb{R}_{\geq 0}} u_{0}\right)\right)(t)+M_{1}\left(\mathcal{L}_{\rho} u\right)(t)+(\mathrm{i} t+\rho) M_{0}\left(\mathcal{L}_{\rho} \chi_{\mathbb{R}_{\geq 0}} u_{0}\right)(t) \\
& =M_{0} \mathcal{L}_{\rho}\left(\partial_{0, \rho}\left(u-\chi_{\mathbb{R}_{\geq 0}} u_{0}\right)\right)(t)+M_{1}\left(\mathcal{L}_{\rho} u\right)(t)+\frac{1}{\sqrt{2 \pi}} M_{0} u_{0} \\
& =\mathcal{L}_{\rho}\left(M_{0} u^{\prime}+M_{1} u\right)(t)+\frac{1}{\sqrt{2 \pi}} M_{0} u_{0} \\
& =\frac{1}{\sqrt{2 \pi}} M_{0} u_{0}
\end{aligned}
$$

from which we read off

$$
\left(\mathcal{L}_{\rho} u\right)(t)=\frac{1}{\sqrt{2 \pi}}\left((\mathrm{i} t+\rho) M_{0}+M_{1}\right)^{-1} M_{0} u_{0}
$$

for each $t \in \mathbb{R}$.

As we have seen in Proposition 5.1, the solution $u$ of (IVP1) is given by the relation

$$
\left(\mathcal{L}_{\rho} u\right)(t)=\frac{1}{\sqrt{2 \pi}}\left((\mathrm{i} t+\rho) M_{0}+M_{1}\right)^{-1} M_{0} u_{0} \quad(t \in \mathbb{R})
$$

for some $\rho>0$ large enough and $u_{0} \in \mathrm{IV}$. Thus, we obtain the following consequence of Corollary 4.5 and Proposition 5.1:

Corollary 5.2. Let $u_{0} \in \mathrm{IV}$. Then for $\rho>0$ large enough we get

$$
\begin{aligned}
\chi_{\mathbb{R}_{\geq 0}}(t) \exp \left(-t\left(\iota_{R\left(M_{0}\right)}^{*} M_{0} \iota_{\mathrm{IV}}\right)^{-1} \iota_{R\left(M_{0}\right)}^{*} M_{1} \iota_{\mathrm{IV}}\right) u_{0} \\
=\frac{1}{\sqrt{2 \pi}}\left(\mathcal{L}_{\rho}^{*}\left((\mathrm{i} \cdot+\rho) M_{0}+M_{1}\right)^{-1} M_{0} u_{0}\right)(t) \quad(t \in \mathbb{R}) .
\end{aligned}
$$


Note that the right-hand side of the equation in Corollary 5.2 also makes sense for $u_{0} \in H$. Indeed, if we just require $u_{0} \in H$, then $u$ given as above solves a weaker variant of the initial value problem (IVP1): We introduce the respective notion first:

Definition 5.3. Let $u_{0} \in H$, and $u: \mathbb{R}_{\geq 0} \rightarrow H$ locally integrable. Then $u$ is a mild solution of (IVP1), if $u$ satisfies

$$
\begin{gathered}
\left(M_{0} u\right)(t)+\int_{0}^{t} M_{1} u(s) \mathrm{d} s=M_{0} u_{0} \quad(t>0), \\
\left(M_{0} u\right)(0+)=M_{0} u_{0} .
\end{gathered}
$$

Note that, if $u$ is a solution to (IVP2 $)$, then it follows from Lebesgue's dominated convergence theorem that $M_{0} u: \mathbb{R}_{\geq 0} \rightarrow H$ is continuous and that the initial datum is attained.

Theorem 5.4. Let $u \in L_{1, \text { loc }}(\mathbb{R} ; H)$. Then the following are equivalent

(i) $\operatorname{spt} u \subseteq \mathbb{R}_{\geq 0}$ and $u$ satisfies

$$
\begin{aligned}
\left(M_{0} u\right)^{\prime}(t)+M_{1} u(t) & =0 \quad(t>0 \text { a.e. }), \\
\lim _{t \rightarrow 0+} \frac{1}{t} \int_{0}^{t}\left|M_{0}\left(u(s)-u_{0}\right)\right| \mathrm{d} s & =0
\end{aligned}
$$

in the sense of distributions, i.e.

$$
-\int_{0}^{\infty}\left\langle M_{0} u(t), \varphi^{\prime}(t)\right\rangle_{H} \mathrm{~d} t+\int_{0}^{\infty}\left\langle M_{1} u(t), \varphi(t)\right\rangle_{H} d t=0
$$

for each $\varphi \in C_{c}^{\infty}\left(\mathbb{R}_{>0} ; H\right)$.

(ii) $\operatorname{spt} u \subseteq \mathbb{R}_{\geq 0}$ and

$$
M_{0} u(t)+\int_{0}^{t} M_{1} u(s) \mathrm{d} s=M_{0} u_{0} \quad(t>0 \text { a.e. })
$$

i.e., $u$ is a mild solution of (IVP1).

(iii) For $\rho>\max \left\{s_{0}(\mathcal{M}), 0\right\}$ we have $u \in L_{2, \rho}(\mathbb{R} ; H)$ and

$$
\left(\mathcal{L}_{\rho} u\right)(t)=\frac{1}{\sqrt{2 \pi}}\left((\mathrm{i} t+\rho) M_{0}+M_{1}\right)^{-1} M_{0} u_{0} \quad(t \in \mathbb{R}) .
$$

In the latter case $M_{0}\left(u-\chi_{\mathbb{R}_{\geq 0}} u_{0}\right) \in H_{\rho}^{1}(\mathbb{R} ; H)$ for each $\rho>\max \left\{s_{0}(\mathcal{M}), 0\right\}$. 
Note that, by Theorem 5.4 we deduce that if $u$ is a mild solution of (IVP1), then $u \in L_{2, \rho}(\mathbb{R} ; H)$. Moreover, we obtain that $M_{0}\left(u-\chi_{\mathbb{R}_{\geq 0}} u_{0}\right) \in H_{\rho}^{1}(\mathbb{R} ; H)$ with

$$
\partial_{0, \rho} M_{0}\left(u-\chi_{\mathbb{R}_{\geq 0}} u_{0}\right)+M_{1} u=0
$$

valid in $L_{2, \rho}(\mathbb{R} ; H)$ for some $\rho>0$. Since the pencil $\mathcal{M}$ associated with $\left(M_{0}, M_{1}\right)$ is regular, we obtain $\left(\partial_{0, \rho} M_{0}+M_{1}\right)^{-1} \in L\left(L_{2, \rho}(\mathbb{R} ; H)\right)$ is well-defined (see e.g. [17, Lemma $2.2])$ and

$$
u=\chi_{\mathbb{R}_{\geq 0}} u_{0}-\left(\partial_{0, \rho} M_{0}+M_{1}\right)^{-1} M_{1} \chi_{\mathbb{R}_{\geq 0}} u_{0},
$$

which yields another representation for the solution $u$.

Before we can come to the proof of Theorem 5.4, we need some auxiliary results first. These statements focus on relating properties of holomorphic functions to the support of their Fourier-Laplace transform. In this sense, one can think of the subsequent assertions as variant of the Paley-Wiener theorem. For this we need a prerequisite:

Lemma 5.5 (see also [16, Lemma 3.6]). Let $\mu, \rho \in \mathbb{R}$ with $\mu<\rho$ and let $U:=$ $\{z \in \mathbb{C} ; \mu<\operatorname{Re} z<\rho\}$. Moreover, let $f: \bar{U} \rightarrow H$ be continuous and analytic in $U$ with

$$
M:=\sup _{z \in \bar{U}}|z f(z)|_{H}<\infty .
$$

Then $f(\mathrm{i} \cdot+\kappa) \in L_{2}(\mathbb{R} ; H)$ for each $\mu \leq \kappa \leq \rho$ and

$$
\left(\mathcal{L}_{\rho}^{*} f(\mathrm{i} \cdot+\rho)\right)(t)=\left(\mathcal{L}_{\mu}^{*} f(\mathrm{i} \cdot+\mu)\right)(t) \quad(t \in \mathbb{R} \text { a.e. }) .
$$

Proof. The first claim follows from the boundedness of $t \mapsto(\mathrm{i} t+\kappa) f(\mathrm{i} t+\kappa)$ for each $\mu \leq \kappa \leq \rho$. For showing the second claim, let $\left(R_{n}\right)_{n \in \mathbb{N}}$ be a sequence in $\mathbb{R}_{>0}$ such that $R_{n} \rightarrow \infty$ and

$$
\begin{aligned}
& \left(\mathcal{L}_{\rho}^{*} \chi_{\left[-R_{n}, R_{n}\right]} f(\mathrm{i} \cdot+\rho)\right)(t) \rightarrow\left(\mathcal{L}_{\rho}^{*} f(\mathrm{i} \cdot+\rho)\right)(t) \\
& \left(\mathcal{L}_{\mu}^{*} \chi_{\left[-R_{n}, R_{n}\right]} f(\mathrm{i} \cdot+\mu)\right)(t) \rightarrow\left(\mathcal{L}_{\mu}^{*} f(\mathrm{i} \cdot+\mu)\right)(t)
\end{aligned}
$$

for almost every $t \in \mathbb{R}$ as $n \rightarrow \infty$. For $n \in \mathbb{N}, t \in \mathbb{R}$ we compute

$$
\begin{aligned}
& \left|\left(\mathcal{L}_{\rho}^{*} \chi_{\left[-R_{n}, R_{n}\right]} f(\mathrm{i} \cdot+\rho)\right)(t)-\left(\mathcal{L}_{\mu}^{*} \chi_{\left[-R_{n}, R_{n}\right]} f(\mathrm{i} \cdot+\mu)\right)(t)\right|_{H} \\
& =\frac{1}{\sqrt{2 \pi}}\left|\int_{R_{n}}^{R_{n}} \mathrm{e}^{(\mathrm{i} s+\rho) t} f(\mathrm{i} s+\rho) \mathrm{d} s-\int_{-R_{n}}^{R_{n}} \mathrm{e}^{(\mathrm{i} s+\mu) t} f(\mathrm{i} s+\mu) \mathrm{d} s\right|_{H} \\
& =\frac{1}{\sqrt{2 \pi}}\left|\int_{\mu}^{\rho} \mathrm{e}^{\left(\mathrm{i} R_{n}+\kappa\right) t} f\left(\mathrm{i} R_{n}+\kappa\right) \mathrm{d} \kappa-\int_{\mu}^{\rho} \mathrm{e}^{\left(-\mathrm{i} R_{n}+\kappa\right) t} f\left(-\mathrm{i} R_{n}+\kappa\right) \mathrm{d} \kappa\right|_{H}
\end{aligned}
$$

by the Cauchy integral theorem. Hence, we can estimate

$$
\left|\left(\mathcal{L}_{\rho}^{*} \chi_{\left[-R_{n}, R_{n}\right]} f(\mathrm{i} \cdot+\rho)\right)(t)-\left(\mathcal{L}_{\mu}^{*} \chi_{\left[-R_{n}, R_{n}\right]} f(\mathrm{i} \cdot+\mu)\right)(t)\right|_{H}
$$




$$
\leq \frac{2 M}{\sqrt{2 \pi}} \int_{\mu}^{\rho} \mathrm{e}^{\kappa t} \frac{1}{\left|\mathrm{i} R_{n}+\kappa\right|} \mathrm{d} \kappa \rightarrow 0 \quad(n \rightarrow \infty) .
$$

Together with (3), this yields the assertion.

Lemma 5.6. Let $\rho_{0} \in \mathbb{R}$ and $f: \mathbb{C}_{\operatorname{Re} \geq \rho_{0}} \rightarrow H$ continuous, analytic in $\mathbb{C}_{\operatorname{Re}>\rho_{0}}$ with

$$
M:=\sup _{z \in \mathbb{C}_{\mathrm{Re} \geq \rho_{0}}}|z f(z)|_{H}<\infty .
$$

Then $\operatorname{spt} \mathcal{L}_{\rho_{0}}^{*} f\left(\mathrm{i} \cdot+\rho_{0}\right) \subseteq \mathbb{R}_{\geq 0}$.

Proof. Let $\varphi \in C_{c}^{\infty}\left(\mathbb{R}_{<0} ; H\right)$ and $\rho>\max \left\{\rho_{0}, 0\right\}$. Using Lemma [5.5] we obtain

$$
\begin{aligned}
& \left|\int_{\mathbb{R}}\left\langle\left(\mathcal{L}_{\rho_{0}}^{*} f\left(\mathrm{i} \cdot+\rho_{0}\right)\right)(t), \varphi(t)\right\rangle_{H} \mathrm{~d} t\right| \\
& =\left|\int_{\mathbb{R}}\left\langle\left(\mathcal{L}_{\rho}^{*} f(\mathrm{i} \cdot+\rho)\right)(t), \varphi(t) \mathrm{e}^{2 \rho t}\right\rangle_{H} \mathrm{e}^{-2 \rho t} \mathrm{~d} t\right| \\
& \leq\left|\mathcal{L}_{\rho}^{*} f(\mathrm{i} \cdot+\rho)\right|_{\rho}\left|\varphi \mathrm{e}^{2 \rho(\cdot)}\right|_{\rho} \\
& =|f(\mathrm{i} \cdot+\rho)|_{0}\left(\int_{-\infty}^{0}\left|\varphi(t) \mathrm{e}^{2 \rho t}\right|_{H}^{2} \mathrm{e}^{-2 \rho t} \mathrm{~d} t\right)^{\frac{1}{2}} \\
& \leq M\left|\frac{1}{\mathrm{i} \cdot+\rho}\right|_{0}|\varphi|_{0} \\
& =M \sqrt{\frac{\pi}{\rho}}|\varphi|_{0} .
\end{aligned}
$$

Letting $\rho$ tend to infinity, we infer

$$
\int_{\mathbb{R}}\left\langle\left(\mathcal{L}_{\rho_{0}}^{*} f\left(\mathrm{i} \cdot+\rho_{0}\right)\right)(t), \varphi(t)\right\rangle_{H} \mathrm{~d} t=0
$$

which yields the assertion.

Remark 5.7. The latter lemma is a special case of the famous Paley-Wiener Theorem (see [8] or [14, 19.2 Theorem]), characterising the $L_{2}$ functions supported on the positive real line by their Laplace transform lying in the Hardy space $\mathcal{H}^{2}\left(\mathbb{C}_{\operatorname{Re}>0} ; H\right)$. However, to keep this article self-contained and since we do not need this deeper result here, we restrict ourselves to the easier case outlined in Lemma 5.6.

Corollary 5.8. Let $\rho_{0} \in \mathbb{R}, F: \mathbb{C}_{\mathrm{Re} \geq \rho_{0}} \rightarrow L(H)$ bounded, continuous and analytic in $\mathbb{C}_{\operatorname{Re}>\rho_{0}}$. Let $g \in L_{2, \rho_{0}}(\mathbb{R} ; H)$ with spt $g \subseteq \mathbb{R}_{\geq a}$ for some $a \in \mathbb{R}$. Then

$$
\operatorname{spt} \mathcal{L}_{\rho_{0}}^{*} F\left(\mathrm{i} \cdot+\rho_{0}\right) \mathcal{L}_{\rho_{0}} g \subseteq \mathbb{R}_{\geq a} .
$$


Proof. Let $\left(\phi_{n}\right)_{n \in \mathbb{N}}$ be a sequence in $C_{c}^{\infty}(\mathbb{R} ; H)$ such that $\operatorname{spt} \phi_{n} \subseteq \mathbb{R}_{\geq a}$ for all $n \in \mathbb{N}$ and $\phi_{n} \rightarrow g$ in $L_{2, \rho_{0}}(\mathbb{R} ; H)$ as $n \rightarrow \infty$. By continuity

$$
\mathcal{L}_{\rho_{0}}^{*} F\left(\mathrm{i} \cdot+\rho_{0}\right) \mathcal{L}_{\rho_{0}} \phi_{n} \rightarrow \mathcal{L}_{\rho_{0}}^{*} F\left(\mathrm{i} \cdot+\rho_{0}\right) \mathcal{L}_{\rho_{0}} g \text { in } L_{2, \rho_{0}}(\mathbb{R} ; H) \quad(n \rightarrow \infty)
$$

and hence, it suffices to show that

$$
\operatorname{spt} \mathcal{L}_{\rho_{0}}^{*} F\left(\mathrm{i} \cdot+\rho_{0}\right) \mathcal{L}_{\rho_{0}} \psi \subseteq \mathbb{R}_{\geq a}
$$

for $\psi \in C_{c}^{\infty}\left(\mathbb{R}_{\geq a} ; H\right)$. Next, we shall argue that we may assume without loss of generality $a=0$. Indeed, let $\psi \in C_{c}^{\infty}\left(\mathbb{R}_{\geq a} ; H\right)$ and consider the function $\widetilde{\psi}:=\psi(\cdot+a) \in$ $C_{c}^{\infty}\left(\mathbb{R}_{\geq 0} ; H\right)$. Then we have

$$
\mathcal{L}_{\rho_{0}} \widetilde{\psi}=\mathrm{e}^{\left(-\mathrm{i}(\cdot)+\rho_{0}\right) a} \mathcal{L}_{\rho_{0}} \psi
$$

and consequently

$$
\left(\mathcal{L}_{\rho_{0}}^{*} F\left(\mathrm{i} \cdot+\rho_{0}\right) \mathcal{L}_{\rho_{0}} \widetilde{\psi}\right)=\left(\mathcal{L}_{\rho_{0}}^{*} F\left(\mathrm{i} \cdot+\rho_{0}\right) \mathcal{L}_{\rho_{0}} \psi\right)(\cdot+a) .
$$

Thus, we need to check

$$
\operatorname{spt} \mathcal{L}_{\rho_{0}}^{*} F\left(\mathrm{i} \cdot+\rho_{0}\right) \mathcal{L}_{\rho_{0}} \widetilde{\psi} \subseteq \mathbb{R}_{\geq 0} .
$$

Hence, proving the case $a=0$ indeed implies the general statement. So, consider the function $f: \mathbb{C}_{\operatorname{Re} \geq \rho_{0}} \rightarrow H$, given by

$$
f(z)=F(z) \widehat{\psi}(z)
$$

where

$$
\widehat{\psi}(z)=\frac{1}{\sqrt{2 \pi}} \int_{\mathbb{R}} \mathrm{e}^{-z s} \psi(s) \mathrm{d} s=\left(\mathcal{L}_{\operatorname{Re} z} \psi\right)(\operatorname{Im} z) .
$$

Then clearly, $f$ is continuous and analytic in $\mathbb{C}_{\operatorname{Re}>\rho_{0}}$ and

$$
|z f(z)|_{H}=\left|F(z) \widehat{\psi}^{\prime}(z)\right|_{H} \leq\|F\|_{\infty}\left\|\psi^{\prime}\right\|_{\infty} \frac{1}{\sqrt{2 \pi}} \int_{0}^{\max \operatorname{spt} \psi} \mathrm{e}^{-\rho_{0} s} \mathrm{~d} s .
$$

Hence,

$$
\operatorname{spt} \mathcal{L}_{\rho_{0}}^{*} F\left(\mathrm{i} \cdot+\rho_{0}\right) \mathcal{L}_{\rho_{0}} \widetilde{\psi}=\operatorname{spt} \mathcal{L}_{\rho_{0}}^{*} f\left(\mathrm{i} \cdot+\rho_{0}\right) \subseteq \mathbb{R}_{\geq 0}
$$

by Lemma 5.6 .

Having these prerequisites at hand, we are now in the position to prove the main result of this section. 
Proof of Theorem 5.4. (i) $\Rightarrow$ (ii): Let $\psi \in C_{c}^{\infty}\left(\mathbb{R}_{\geq 0} ; H\right)$. We compute

$$
\begin{aligned}
\int_{0}^{\infty}\left\langle M_{0} u(t)+\int_{0}^{t} M_{1} u(s) \mathrm{d} s, \psi(t)\right\rangle & \mathrm{d} t \\
& =\int_{0}^{\infty}\left\langle M_{0} u(t), \psi(t)\right\rangle_{H} \mathrm{~d} t+\int_{0}^{\infty}\left\langle M_{1} u(t), \int_{t}^{\infty} \psi(s) \mathrm{d} s\right\rangle_{H} \mathrm{~d} t .
\end{aligned}
$$

Setting $\varphi(t):=\int_{t}^{\infty} \psi(s) \mathrm{d} s$ for $t \in \mathbb{R}_{\geq 0}$, the latter can be expressed by

$$
\begin{aligned}
\int_{0}^{\infty}\left\langle M_{0} u(t)+\int_{0}^{t} M_{1} u(s) \mathrm{d} s, \psi(t)\right\rangle & \mathrm{d} t \\
& =-\int_{0}^{\infty}\left\langle M_{0} u(t), \varphi^{\prime}(t)\right\rangle_{H} \mathrm{~d} t+\int_{0}^{\infty}\left\langle M_{1} u(t), \varphi(t)\right\rangle_{H} \mathrm{~d} t .
\end{aligned}
$$

Let now $\eta_{n} \in C^{\infty}(\mathbb{R})$ such that spt $\eta_{n} \subseteq\left[\frac{1}{n}, \infty\left[, \eta_{n}=1\right.\right.$ on $\left[\frac{2}{n}, \infty\left[\right.\right.$ and $\left|\eta_{n}\right|_{\infty} \leq 1,\left|\eta_{n}^{\prime}\right|_{\infty} \leq$ $2 n$ for each $n \in \mathbb{N}$. Set $\varphi_{n}:=\varphi \eta_{n} \in C_{c}^{\infty}\left(\mathbb{R}_{>0} ; H\right)$ for $n \in \mathbb{N}$. We obtain

$$
\begin{aligned}
& -\int_{0}^{\infty}\left\langle M_{0} u(t), \varphi^{\prime}(t)\right\rangle_{H} \mathrm{~d} t+\int_{0}^{\infty}\left\langle M_{1} u(t), \varphi(t)\right\rangle_{H} \mathrm{~d} t \\
= & \lim _{n \rightarrow \infty}\left(-\int_{0}^{\infty}\left\langle M_{0} u(t), \varphi^{\prime}(t) \eta_{n}(t)\right\rangle_{H} \mathrm{~d} t+\int_{0}^{\infty}\left\langle M_{1} u(t), \varphi_{n}(t)\right\rangle_{H} \mathrm{~d} t\right) \\
= & \lim _{n \rightarrow \infty}\left(-\int_{0}^{\infty}\left\langle M_{0} u(t), \varphi_{n}^{\prime}(t)\right\rangle_{H} \mathrm{~d} t+\int_{0}^{\infty}\left\langle M_{0} u(t), \varphi(t) \eta_{n}^{\prime}(t)\right\rangle_{H} \mathrm{~d} t+\int_{0}^{\infty}\left\langle M_{1} u(t), \varphi_{n}(t)\right\rangle_{H} \mathrm{~d} t\right) \\
= & \lim _{n \rightarrow \infty} \int_{0}^{\infty}\left\langle M_{0} u(t), \varphi(t) \eta_{n}^{\prime}(t)\right\rangle_{H} \mathrm{~d} t .
\end{aligned}
$$

Moreover, we have that

$$
\left|\int_{0}^{\infty}\left\langle M_{0}\left(u(t)-u_{0}\right), \varphi(t) \eta_{n}^{\prime}(t)\right\rangle_{H} \mathrm{~d} t\right| \leq 2 n\|\varphi\|_{\infty} \int_{0}^{\frac{2}{n}}\left|M_{0}\left(u(t)-u_{0}\right)\right|_{H} \mathrm{~d} t \rightarrow 0 \quad(n \rightarrow \infty)
$$

and thus,

$$
\lim _{n \rightarrow \infty} \int_{0}^{\infty}\left\langle M_{0} u(t), \varphi(t) \eta_{n}^{\prime}(t)\right\rangle_{H} \mathrm{~d} t
$$




$$
\begin{aligned}
& =\lim _{n \rightarrow \infty} \int_{0}^{\infty}\left\langle M_{0} u_{0}, \varphi(t) \eta_{n}^{\prime}(t)\right\rangle_{H} \mathrm{~d} t \\
& =\lim _{n \rightarrow \infty}\left(\int_{0}^{\infty}\left\langle M_{0} u_{0}, \varphi_{n}^{\prime}(t)\right\rangle_{H} \mathrm{~d} t-\int_{0}^{\infty}\left\langle M_{0} u_{0}, \varphi^{\prime}(t) \eta_{n}(t)\right\rangle_{H} \mathrm{~d} t\right) \\
& =-\int_{0}^{\infty}\left\langle M_{0} u_{0}, \varphi^{\prime}(t)\right\rangle_{H} \mathrm{~d} t \\
& =\int_{0}^{\infty}\left\langle M_{0} u_{0}, \psi(t)\right\rangle_{H} \mathrm{~d} t .
\end{aligned}
$$

Summarising, we have shown that

$$
\int_{0}^{\infty}\left\langle M_{0} u(t)+\int_{0}^{t} M_{1} u(s) \mathrm{d} s, \psi(t)\right\rangle_{H} \mathrm{~d} t=\int_{0}^{\infty}\left\langle M_{0} u_{0}, \psi(t)\right\rangle_{H} \mathrm{~d} t
$$

for each $\psi \in C_{c}^{\infty}\left(\mathbb{R}_{\geq 0} ; H\right)$ and hence, the assertion follows.

(ii) $\Rightarrow$ (iii): Let $\varphi \in C_{c}^{\infty}(\mathbb{R} ; H)$ and $\rho>\max \left\{s_{0}(\mathcal{M}), 0\right\}$. Denote by $a:=\max \operatorname{spt} \varphi$. We set

$$
\psi(t):=(-\mathrm{i} t+\rho)\left((-\mathrm{i} t+\rho) M_{0}^{*}+M_{1}^{*}\right)^{-1}\left(\mathcal{L}_{\rho} \varphi\right)(t) \quad(t \in \mathbb{R}) .
$$

Then we infer

$$
\begin{aligned}
\int_{\mathbb{R}}\langle u(t), \varphi(t)\rangle_{H} \mathrm{e}^{-2 \rho t} \mathrm{~d} t & =\int_{0}^{a}\langle u(t), \varphi(t)\rangle_{H} \mathrm{e}^{-2 \rho t} \mathrm{~d} t \\
& =\int_{0}^{a}\left\langle u(t),\left(\mathcal{L}_{\rho}^{*}\left(M_{0}^{*}+\frac{1}{-\mathrm{i} \cdot+\rho} M_{1}^{*}\right) \psi\right)(t)\right\rangle_{H} \mathrm{e}^{-2 \rho t} \mathrm{~d} t .
\end{aligned}
$$

As a first step we show that spt $\left.\left.\mathcal{L}_{\rho}^{*} \psi \subseteq\right]-\infty, a\right]$. For doing so, let $\xi \in C_{c}^{\infty}\left(\mathbb{R}_{>a}, H\right)$. Then we compute

$$
\begin{aligned}
\int_{\mathbb{R}}\left\langle\mathcal{L}_{\rho}^{*} \psi(t), \xi(t)\right\rangle_{H} \mathrm{e}^{-2 \rho t} \mathrm{~d} t & =\int_{\mathbb{R}}\left\langle\psi(t), \mathcal{L}_{\rho} \xi(t)\right\rangle_{H} \mathrm{~d} t \\
& =\int_{\mathbb{R}}\left\langle\left(\mathcal{L}_{\rho} \varphi\right)(t),\left((\mathrm{i} t+\rho) M_{0}+M_{1}\right)^{-1}\left(\mathcal{L}_{\rho} \xi^{\prime}\right)(t)\right\rangle_{H} \mathrm{~d} t \\
& =\int_{-\infty}^{a}\left\langle\varphi(t),\left(\mathcal{L}_{\rho}^{*}\left((\mathrm{i} \cdot+\rho) M_{0}+M_{1}\right)^{-1} \mathcal{L}_{\rho} \xi^{\prime}\right)(t)\right\rangle_{H} \mathrm{e}^{-2 \rho t} \mathrm{~d} t,
\end{aligned}
$$


where in the last equality we used $\operatorname{spt} \varphi \subseteq]-\infty, a]$. Next, by Corollary 5.8 applied to $g=$ $\xi^{\prime}$ and $F: z \mapsto\left(z M_{0}+M_{1}\right)^{-1}$, we deduce that spt $\xi^{\prime} \subseteq \mathbb{R}_{\geq a} \operatorname{implies} \operatorname{spt}\left(\mathcal{L}_{\rho}^{*} F(\mathrm{i} \cdot+\rho) \mathcal{L}_{\rho} \xi^{\prime}\right) \subseteq$ $\mathbb{R}_{\geq a}$. Hence,

$$
\int_{\mathbb{R}}\left\langle\mathcal{L}_{\rho}^{*} \psi(t), \xi(t)\right\rangle_{H} \mathrm{e}^{-2 \rho t} \mathrm{~d} t=0 .
$$

Since $\xi$ was arbitrary, we conclude the first step and deduce that

$$
\left.\left.\operatorname{spt} \mathcal{L}_{\rho}^{*} \psi \subseteq\right]-\infty, a\right] .
$$

Moreover, we compute

$$
\begin{aligned}
\mathcal{L}_{\rho}^{*}\left(\frac{1}{-\mathrm{i} \cdot+\rho} \psi\right)(t) \mathrm{e}^{-2 \rho t} & =\frac{1}{\sqrt{2 \pi}} \int_{\mathbb{R}} \frac{1}{-\mathrm{i} s+\rho} \psi(s) \mathrm{e}^{(\mathrm{i} s-\rho) t} \mathrm{~d} s \\
& =\frac{1}{\sqrt{2 \pi}} \int_{\mathbb{R}} \int_{t}^{\infty} \mathrm{e}^{(\mathrm{i} s-\rho) r} \mathrm{~d} r \psi(s) \mathrm{d} s \\
& =\int_{t}^{\infty} \mathrm{e}^{-\rho r} \frac{1}{\sqrt{2 \pi}} \int_{\mathbb{R}} \mathrm{e}^{\mathrm{i} s r} \psi(s) \mathrm{d} s \mathrm{~d} r \\
& =\int_{t}^{a}\left(\mathcal{L}_{\rho}^{*} \psi\right)(r) \mathrm{e}^{-2 \rho r} \mathrm{~d} r
\end{aligned}
$$

for each $t \in \mathbb{R}$ and hence, with (4) and (6) we get

$$
\begin{aligned}
& \int_{\mathbb{R}}\langle u(t), \varphi(t)\rangle_{H} \mathrm{e}^{-2 \rho t} \mathrm{~d} t \\
= & \int_{0}^{a}\left\langle u(t), M_{0}^{*}\left(\mathcal{L}_{\rho}^{*} \psi\right)(t) \mathrm{e}^{-2 \rho t}+M_{1}^{*} \int_{t}^{a}\left(\mathcal{L}_{\rho}^{*} \psi\right)(r) \mathrm{e}^{-2 \rho r} \mathrm{~d} r\right\rangle_{H} \mathrm{~d} t \\
= & \int_{0}^{a}\left\langle M_{0} u(t),\left(\mathcal{L}_{\rho}^{*} \psi\right)(t)\right\rangle_{H} \mathrm{e}^{-2 \rho t} \mathrm{~d} t+\int_{0}^{a} \int_{t}^{a}\left\langle M_{1} u(t),\left(\mathcal{L}_{\rho}^{*} \psi\right)(r)\right\rangle_{H} \mathrm{e}^{-2 \rho r} \mathrm{~d} r \mathrm{~d} t \\
= & \int_{0}^{a}\left\langle M_{0} u(t),\left(\mathcal{L}_{\rho}^{*} \psi\right)(t)\right\rangle_{H} \mathrm{e}^{-2 \rho t} \mathrm{~d} t+\int_{0}^{a}\left\langle\int_{0}^{r} M_{1} u(t) \mathrm{d} t,\left(\mathcal{L}_{\rho}^{*} \psi\right)(r)\right\rangle_{H} \mathrm{e}^{-2 \rho r} \mathrm{~d} r \\
= & \int_{0}^{a}\left\langle M_{0} u(t)+\int_{0}^{t} M_{1} u(r) \mathrm{d} r,\left(\mathcal{L}_{\rho}^{*} \psi\right)(t)\right\rangle_{H} \mathrm{e}^{-2 \rho t} \mathrm{~d} t .
\end{aligned}
$$

By using (ii) and (5), we further obtain

$$
\int_{\mathbb{R}}\langle u(t), \varphi(t)\rangle_{H} \mathrm{e}^{-2 \rho t} \mathrm{~d} t
$$




$$
\begin{aligned}
& \int_{0}^{a}\left\langle M_{0} u_{0},\left(\mathcal{L}_{\rho}^{*} \psi\right)(t)\right\rangle_{H} \mathrm{e}^{-2 \rho t} \mathrm{~d} t \\
= & \left\langle\chi_{\mathbb{R}_{\geq 0}} M_{0} u_{0}, \mathcal{L}_{\rho}^{*} \psi\right\rangle_{\rho} \\
= & \left\langle\mathcal{L}_{\rho} \chi_{\mathbb{R}_{\geq 0}} M_{0} u_{0}, \psi\right\rangle_{0} \\
= & \frac{1}{\sqrt{2 \pi}} \int_{\mathbb{R}}\left\langle\frac{1}{\mathrm{i} t+\rho} M_{0} u_{0},(-\mathrm{i} t+\rho)\left((-\mathrm{i} t+\rho) M_{0}^{*}+M_{1}^{*}\right)^{-1}\left(\mathcal{L}_{\rho} \varphi\right)(t)\right\rangle_{H} \mathrm{~d} t \\
= & \frac{1}{\sqrt{2 \pi}} \int_{\mathbb{R}}\left\langle\left((\mathrm{i} t+\rho) M_{0}+M_{1}\right)^{-1} M_{0} u_{0},\left(\mathcal{L}_{\rho} \varphi\right)(t)\right\rangle_{H} \mathrm{~d} t \\
= & \left\langle\mathcal{L}_{\rho}^{*} \frac{1}{\sqrt{2 \pi}}\left((\mathrm{i} \cdot+\rho) M_{0}+M_{1}\right)^{-1} M_{0} u_{0}, \varphi\right\rangle_{\rho} .
\end{aligned}
$$

Since $\varphi \in C_{c}^{\infty}(\mathbb{R} ; H)$ was chosen arbitrarily, we obtain (iii).

(iii) $\Rightarrow$ (i): Consider the function

$$
f(z)=\left(z M_{0}+M_{1}\right)^{-1} M_{0} u_{0}=\frac{1}{z} M_{0} u_{0}+\frac{1}{z}\left(z M_{0}+M_{1}\right)^{-1} M_{1} u_{0} \quad\left(z \in \mathbb{C}_{\operatorname{Re} \geq \rho}\right) .
$$

Then, $f$ is continuous and analytic in $\mathbb{C}_{\operatorname{Re}>\rho}$ and satisfies

$$
|z f(z)| \leq\left(\left\|M_{0}\right\|+\left\|\mathcal{M}(z)^{-1}\right\|\left\|M_{1}\right\|\right)\left|u_{0}\right| .
$$

Thus, by Lemma 5.6 we infer that

$$
u=\frac{1}{\sqrt{2 \pi}} \mathcal{L}_{\rho}^{*}\left((\mathrm{i} \cdot+\rho) M_{0}+M_{1}\right)^{-1} M_{0} u_{0}=\frac{1}{\sqrt{2 \pi}} \mathcal{L}_{\rho}^{*} f(\mathrm{i} \cdot+\rho)
$$

is supported on $\mathbb{R}_{\geq 0}$. Moreover, we compute

$$
\begin{aligned}
(\mathrm{i} t+\rho)\left(\mathcal{L}_{\rho} M_{0}\left(u-\chi_{\mathbb{R}_{\geq 0}} u_{0}\right)\right)(t) & =(\mathrm{i} t+\rho) M_{0}\left(\mathcal{L}_{\rho} u\right)(t)-\frac{1}{\sqrt{2 \pi}} M_{0} u_{0} \\
& =\frac{1}{\sqrt{2 \pi}}\left((\mathrm{i} t+\rho) M_{0}\left((\mathrm{i} t+\rho) M_{0}+M_{1}\right)^{-1}-1\right) M_{0} u_{0} \\
& =-\frac{1}{\sqrt{2 \pi}} M_{1}\left((\mathrm{i} t+\rho) M_{0}+M_{1}\right)^{-1} M_{0} u_{0} \\
& =-M_{1}\left(\mathcal{L}_{\rho} u\right)(t)
\end{aligned}
$$

for each $t \in \mathbb{R}$. Hence, since $M_{1} \mathcal{L}_{\rho} u \in L_{2}(\mathbb{R} ; H)$ we get $M_{0}\left(u-\chi_{\mathbb{R}_{\geq 0}} u_{0}\right) \in H_{\rho}^{1}(\mathbb{R} ; H)$. Therefore, $M_{0}\left(u-\chi_{\mathbb{R}_{\geq 0}} u_{0}\right)$ is continuous by Proposition 2.5 and thus,

$$
\lim _{t \rightarrow 0+} M_{0}\left(u(t)-u_{0}\right)=0,
$$

since $u$ is supported on $\mathbb{R}_{\geq 0}$. In particular, by the fundamental theorem of calculus, we have

$$
\lim _{t \rightarrow 0+} \frac{1}{t} \int_{0}^{t}\left|M_{0}\left(u(s)-u_{0}\right)\right|=0 .
$$


For showing the remaining claim, let $\varphi \in C_{c}^{\infty}\left(\mathbb{R}_{>0}\right)$. From (7) we read off with the help of Proposition 2.2

$$
\partial_{0, \rho} M_{0}\left(u-\chi_{\mathbb{R}_{>0}} u_{0}\right)+M_{1} u=0
$$

and consequently,

$$
\begin{aligned}
& -\int_{0}^{\infty}\left\langle M_{0} u(t), \varphi^{\prime}(t)\right\rangle_{H} \mathrm{~d} t+\int_{0}^{\infty}\left\langle M_{1} u(t), \varphi(t)\right\rangle_{H} \mathrm{~d} t \\
& =-\int_{0}^{\infty}\left\langle M_{0}\left(u(t)-u_{0}\right), \varphi^{\prime}(t)\right\rangle_{H} \mathrm{~d} t+\int_{0}^{\infty}\left\langle M_{1} u(t), \varphi(t)\right\rangle_{H} \mathrm{~d} t \\
& =-\left\langle M_{0}\left(u-\chi_{\mathbb{R}_{\geq 0}} u_{0}\right), \varphi^{\prime} \mathrm{e}^{2 \rho(\cdot)}\right\rangle_{\rho}+\left\langle M_{1} u, \varphi \mathrm{e}^{2 \rho(\cdot)}\right\rangle_{\rho} \\
& =\left\langle M_{0}\left(u-\chi_{\mathbb{R}_{\geq 0}} u_{0}\right),-\left(\varphi \mathrm{e}^{2 \rho(\cdot)}\right)^{\prime}+2 \rho \varphi \mathrm{e}^{2 \rho(\cdot)}\right\rangle_{\rho}+\left\langle M_{1} u, \varphi \mathrm{e}^{2 \rho(\cdot)}\right\rangle_{\rho} \\
& =\left\langle M_{0}\left(u-\chi_{\mathbb{R}_{\geq 0}} u_{0}\right), \partial_{0, \rho}^{*}\left(\varphi \mathrm{e}^{2 \rho(\cdot)}\right)\right\rangle_{\rho}+\left\langle M_{1} u, \varphi \mathrm{e}^{2 \rho(\cdot)}\right\rangle_{\rho} \\
& =0
\end{aligned}
$$

In the following, we shall address the asymptotic properties of the solution $u$, if considered as a solution to (IVP1) or to (IVP2). For this, we associate a spectrum to (IVP1) and (IVP2). The next section proves equality of the spectra to be introduced.

\section{$6 \sigma_{\mathrm{IV}}(\mathcal{M})=\sigma(\mathcal{M})$.}

In this section, we will assume throughout that $\mathcal{M}$ is a regular linear operator pencil associated with $\left(M_{0}, M_{1}\right)$ in some Hilbert space with $R\left(M_{0}\right) \subseteq H$ closed. We consider the following two spectra

$$
\begin{aligned}
\sigma_{\mathrm{IV}}(\mathcal{M}) & =\sigma\left(-\left(\iota_{R\left(M_{0}\right)}^{*} M_{0} \iota_{\mathrm{IV}}\right)^{-1}\left(\iota_{R\left(M_{0}\right)}^{*} M_{1} \iota_{\mathrm{IV}}\right)\right), \\
\sigma(\mathcal{M}) & =\left\{z \in \mathbb{C} ; 0 \in \sigma\left(z M_{0}+M_{1}\right)\right\} .
\end{aligned}
$$

We note that the first spectrum is strongly related to the asymptotics of the initial value problem treated in Section 4 , while the second spectrum determines the asymptotic behaviour of the initial value problem studied in Section 5 (for details see Section 7 ). Before we address the spectra just introduced, we elaborate a bit more on the connection of IV and $N\left(M_{0}\right)^{\perp}$, see also Remark 4.4, We reformulate IV first:

Lemma 6.1. Let $u \in H$. Then $u \in \mathrm{IV}$ if, and only if,

$$
\iota_{N\left(M_{0}\right)}^{*} u=-\left(\iota_{R\left(M_{0}\right)^{\perp}}^{*} M_{1} \iota_{N\left(M_{0}\right)}\right)^{-1} \iota_{R\left(M_{0}\right)^{\perp}}^{*} M_{1} \iota_{N\left(M_{0}\right)^{\perp} \iota_{N\left(M_{0}\right)^{\perp}}^{*} u .}
$$

Proof. With the unitary operators $U_{0}$ and $U_{1}$ as introduced in Proposition 3.2, we have

$$
U_{1} M_{1} U_{0}^{*}=\left(\begin{array}{cc}
\iota_{R\left(M_{0}\right)}^{*} M_{1} \iota_{N\left(M_{0}\right)^{\perp}} & \iota_{R\left(M_{0}\right)}^{*} M_{1} \iota_{N\left(M_{0}\right)} \\
\iota_{R\left(M_{0}\right)^{\perp}}^{*} M_{1} \iota_{N\left(M_{0}\right)^{\perp}} & \iota_{R\left(M_{0}\right)^{\perp}}^{*} M_{1} \iota_{N\left(M_{0}\right)}
\end{array}\right),
$$




$$
U_{1} M_{0} U_{0}^{*}=\left(\begin{array}{cc}
\iota_{R\left(M_{0}\right)}^{*} M_{0} \iota_{N\left(M_{0}\right)^{\perp}} & 0 \\
0 & 0
\end{array}\right) .
$$

Thus, for $u \in H$ with $(x, y):=U_{0} u$ we obtain

$$
\begin{aligned}
& u \in \mathrm{IV} \Longleftrightarrow \exists v \in H: M_{1} u=M_{0} v \\
& \Longleftrightarrow \exists v \in H: U_{1} M_{1} U_{0}^{*} U_{0} u=U_{1} M_{0} U_{0}^{*} U_{0} v \\
& \Longleftrightarrow \exists v \in H:\left(\begin{array}{cc}
\iota_{R\left(M_{0}\right)}^{*} M_{1} \iota_{N\left(M_{0}\right)^{\perp}} & \iota_{R\left(M_{0}\right)}^{*} M_{1} \iota_{N\left(M_{0}\right)} \\
\iota_{R\left(M_{0}\right)^{\perp}}^{*} M_{1} \iota_{N\left(M_{0}\right)^{\perp}} & \iota_{R\left(M_{0}\right)^{\perp}}^{*} M_{1} \iota_{N\left(M_{0}\right)}
\end{array}\right)\left(\begin{array}{c}
x \\
y
\end{array}\right) \\
& =\left(\begin{array}{c}
\iota_{R\left(M_{0}\right)}^{*} M_{0} \iota_{N\left(M_{0}\right)^{\perp} \iota_{N\left(M_{0}\right)^{\perp}}^{*} v} \\
0
\end{array}\right) \\
& \Longleftrightarrow \exists v \in H:\left(\begin{array}{c}
\iota_{R\left(M_{0}\right)}^{*} M_{1} \iota_{N\left(M_{0}\right)^{\perp}} x+\iota_{R\left(M_{0}\right)}^{*} M_{1} \iota_{N\left(M_{0}\right)} y \\
\iota_{R\left(M_{0}\right)^{\perp}}^{*} M_{1} \iota_{N\left(M_{0}\right)^{\perp}} x+\iota_{R\left(M_{0}\right)^{\perp}}^{*} M_{1} \iota_{N\left(M_{0}\right)} y
\end{array}\right) \\
& =\left(\begin{array}{c}
\iota_{R\left(M_{0}\right)}^{*} M_{0} \iota_{N\left(M_{0}\right)^{\perp} \iota_{N\left(M_{0}\right)^{\perp}}^{*} v} v \\
0
\end{array}\right) \\
& \Longleftrightarrow \iota_{R\left(M_{0}\right)^{\perp}}^{*} M_{1} \iota_{N\left(M_{0}\right)^{\perp}} x+\iota_{R\left(M_{0}\right)^{\perp}}^{*} M_{1} \iota_{N\left(M_{0}\right)} y=0,
\end{aligned}
$$

where in the last equivalence we have used the invertibility of $\iota_{R\left(M_{0}\right)}^{*} M_{0} \iota_{N\left(M_{0}\right)^{\perp}}$. Since, $\mathcal{M}$ is regular and $R\left(M_{0}\right) \subseteq H$ is closed, we obtain the assertion by Proposition 3.2. Indeed, an application of Proposition 3.2 yields that $\iota_{R\left(M_{0}\right)^{\perp}}^{*} M_{1} \iota_{N\left(M_{0}\right)}$ is an isomorphism. Thus, for $U_{0} u=\left(\iota_{N\left(M_{0}\right)^{\perp}}^{*} u, \iota_{N\left(M_{0}\right)}^{*} u\right)=(x, y)$ we have

$$
u \in \mathrm{IV} \Longleftrightarrow y=-\left(\iota_{R\left(M_{0}\right)^{\perp}}^{*} M_{1} \iota_{N\left(M_{0}\right)}\right)^{-1} \iota_{R\left(M_{0}\right)^{\perp}}^{*} M_{1} \iota_{N\left(M_{0}\right)^{\perp}} x .
$$

Proposition 6.2. (a) We have $N\left(M_{0}\right)^{\perp} \subseteq$ IV if, and only if, $\iota_{R\left(M_{0}\right)^{\perp}} M_{1} \iota_{N\left(M_{0}\right)^{\perp}}=0$. In either case we have $N\left(M_{0}\right)^{\perp}=\mathrm{IV}$.

(b) We have IV $\cap N\left(M_{0}\right)^{\perp}=\{0\}$ if, and only if, $\iota_{R\left(M_{0}\right)^{\perp}}^{*} M_{1} \iota_{N\left(M_{0}\right)^{\perp}}$ is injective.

Proof. We shall prove (a) first. Using Lemma 6.1 we observe

$$
\begin{aligned}
N\left(M_{0}\right)^{\perp} \subseteq \mathrm{IV} & \Longleftrightarrow \forall x \in N\left(M_{0}\right)^{\perp}:\left(\iota_{R\left(M_{0}\right)^{\perp}}^{*} M_{1} \iota_{N\left(M_{0}\right)}\right)^{-1} \iota_{R\left(M_{0}\right)^{\perp}}^{*} M_{1} \iota_{N\left(M_{0}\right)^{\perp}} x=0 \\
& \Longleftrightarrow \iota_{R\left(M_{0}\right)^{\perp}}^{*} M_{1} \iota_{N\left(M_{0}\right)^{\perp}}=0,
\end{aligned}
$$

which shows the asserted equivalence. Moreover, if $N\left(M_{0}\right)^{\perp} \subseteq \mathrm{IV}$, we infer

$$
\left(\iota_{R\left(M_{0}\right)}^{*} M_{0} \iota_{\mathrm{IV}}\right)^{-1} \iota_{R\left(M_{0}\right)}^{*} M_{0} \iota_{N\left(M_{0}\right)^{\perp}}=1_{N\left(M_{0}\right)^{\perp}},
$$

where we used that by Proposition $4.3 \iota_{R\left(M_{0}\right)}^{*} M_{0} \iota_{\mathrm{IV}}$ is an isomorphism. As

$$
\left(\iota_{R\left(M_{0}\right)}^{*} M_{0} \iota_{\mathrm{IV}}\right)^{-1} \iota_{R\left(M_{0}\right)}^{*} M_{0} \iota_{N\left(M_{0}\right)^{\perp}}
$$


is an isomorphism mapping $N\left(M_{0}\right)^{\perp}$ onto IV (see Remark 4.4), we deduce $N\left(M_{0}\right)^{\perp}=$ IV. For the proof of $(\mathrm{b})$, we observe that by Lemma 6.1

$$
\begin{aligned}
x \in \mathrm{IV} \cap N\left(M_{0}\right)^{\perp} & \Longleftrightarrow x \in N\left(M_{0}\right)^{\perp} \wedge\left(\iota_{R\left(M_{0}\right)^{\perp}}^{*} M_{1} \iota_{N\left(M_{0}\right)}\right)^{-1} \iota_{R\left(M_{0}\right)^{\perp}}^{*} M_{1} \iota_{N\left(M_{0}\right)^{\perp}} x=0 \\
& \Longleftrightarrow x \in \operatorname{ker}\left(\iota_{R\left(M_{0}\right)^{\perp}}^{*} M_{1} \iota_{N\left(M_{0}\right)^{\perp}}\right) .
\end{aligned}
$$

Hence, IV $\cap N\left(M_{0}\right)^{\perp}=\{0\}$ if and only if $\iota_{R\left(M_{0}\right)^{\perp}}^{*} M_{1} \iota_{N\left(M_{0}\right)^{\perp}}$ is one-to-one.

Theorem 6.3. We have $\sigma_{\mathrm{IV}}(\mathcal{M})=\sigma(\mathcal{M})$.

Proof. Recall from Proposition 3.2 that

$$
\sigma(\mathcal{M})=\sigma\left(-\widetilde{M}_{0}^{-1} \widetilde{M}_{1}\right)
$$

where

$$
\widetilde{M}_{0}=\iota_{R\left(M_{0}\right)}^{*} M_{0} \iota_{N\left(M_{0}\right)^{\perp}}
$$

and

$$
\widetilde{M}_{1}=\iota_{R\left(M_{0}\right)}^{*} M_{1} \iota_{N\left(M_{0}\right)^{\perp}}-\iota_{R\left(M_{0}\right)}^{*} M_{1} \iota_{N\left(M_{0}\right)}\left(\iota_{R\left(M_{0}\right)^{\perp}}^{*} M_{1} \iota_{N\left(M_{0}\right)}\right)^{-1} \iota_{R\left(M_{0}\right)^{\perp}} M_{1} \iota_{N\left(M_{0}\right)^{\perp}} .
$$

By definition, we have

$$
\sigma_{\mathrm{IV}}(\mathcal{M})=\sigma\left(-\left(\iota_{R\left(M_{0}\right)}^{*} M_{0} \iota_{\mathrm{IV}}\right)^{-1} \iota_{R\left(M_{0}\right)}^{*} M_{1} \iota_{\mathrm{IV}}\right) .
$$

For $z \in \mathbb{C}$ we show $z \widetilde{M}_{0}+\widetilde{M}_{1}$ is continuously invertible if, and only if,

$$
z \iota_{R\left(M_{0}\right)}^{*} M_{0} \iota_{\mathrm{IV}}+\iota_{R\left(M_{0}\right)}^{*} M_{1} \iota_{\mathrm{IV}} \text { is continuously invertible. }
$$

For this, let $u \in R\left(M_{0}\right)$ and $v \in \mathrm{IV}$ be such that

$$
z \iota_{R\left(M_{0}\right)}^{*} M_{0} \iota_{\mathrm{IV}} v+\iota_{R\left(M_{0}\right)}^{*} M_{1} \iota_{\mathrm{IV}} v=u .
$$

The latter is the same as saying

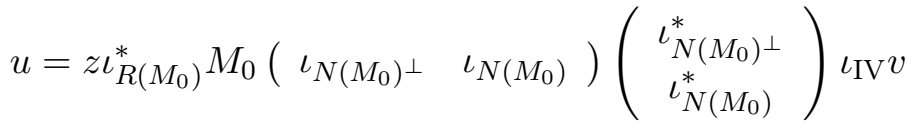

$$
\begin{aligned}
& +\iota_{R\left(M_{0}\right)}^{*} M_{1}\left(\iota_{N\left(M_{0}\right)^{\perp}} \iota_{N\left(M_{0}\right)}\right)\left(\begin{array}{c}
\iota_{N\left(M_{0}\right)^{\perp}}^{*} \\
\iota_{N\left(M_{0}\right)}^{*}
\end{array}\right) \iota_{\mathrm{IV} V} v
\end{aligned}
$$

By Lemma 6.1, we deduce that the latter can be written as

$$
u=\left(\begin{array}{ll}
z \iota_{R\left(M_{0}\right)}^{*} M_{0} \iota_{N\left(M_{0}\right)^{\perp}} & 0
\end{array}\right)\left(\begin{array}{c}
\iota_{N\left(M_{0}\right)^{\perp}}^{*} v \\
-\left(\iota_{R\left(M_{0}\right)^{\perp}}^{*} M_{1} \iota_{N\left(M_{0}\right)}\right)^{-1} \iota_{R\left(M_{0}\right)^{\perp}}^{*} M_{1} \iota_{N\left(M_{0}\right)^{\perp} \iota_{N\left(M_{0}\right)^{\perp}}^{*} v}
\end{array}\right)
$$




$$
\begin{aligned}
& +\left(\iota_{R\left(M_{0}\right)}^{*} M_{1} \iota_{N\left(M_{0}\right)^{\perp}} \quad \iota_{R\left(M_{0}\right)}^{*} M_{1} \iota_{N\left(M_{0}\right)}\right) \times
\end{aligned}
$$

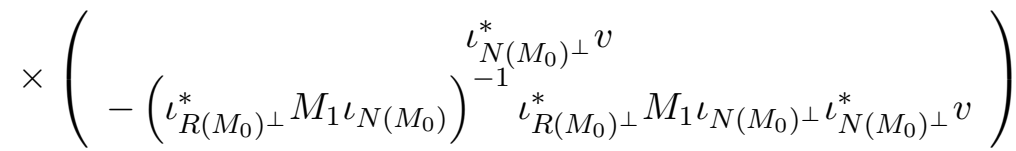

$$
\begin{aligned}
& =z \widetilde{M}_{0} \iota_{N\left(M_{0}\right)^{\perp}}^{*} v+\widetilde{M}_{1} \iota_{N\left(M_{0}\right)^{\perp}}^{*} v .
\end{aligned}
$$

Hence, for $u \in R\left(M_{0}\right)$ and $v \in I V$ the equation

$$
z \iota_{R\left(M_{0}\right)}^{*} M_{0} \iota_{\mathrm{IV}} v+\iota_{R\left(M_{0}\right)}^{*} M_{1} \iota_{\mathrm{IV}} v=u
$$

implies

$$
z \widetilde{M}_{0} \iota_{N\left(M_{0}\right)^{\perp}}^{*} v+\widetilde{M}_{1} \iota_{N\left(M_{0}\right)^{\perp}}^{*} v=u .
$$

On the other hand, following the argument in reverse direction, we get that if $x \in$ $N\left(M_{0}\right)^{\perp}$ solves (10), then

$$
v:=\left(\begin{array}{ll}
\iota_{N\left(M_{0}\right)^{\perp}} & \iota_{N\left(M_{0}\right)}
\end{array}\right)\left(\begin{array}{c}
\iota_{N\left(M_{0}\right)^{\perp}}^{*} x \\
\left.-\left(\iota_{R\left(M_{0}\right)^{\perp}}^{*} M_{1} \iota_{N\left(M_{0}\right)}\right)^{-1} \iota_{R\left(M_{0}\right)^{\perp}}^{*} M_{1} \iota_{N\left(M_{0}\right)^{\perp} \iota_{N\left(M_{0}\right)^{\perp}}^{*} x}^{*}\right)
\end{array}\right.
$$

solves (9). Note that $v \in \mathrm{IV}$, by Lemma 6.1. The assertion follows.

In the next section we discuss the asymptotic properties of the solutions of both initial value problems (IVP1) and (IVP2).

\section{Asymptotic Properties}

In the previous section, we have shown that the spectra connected to strong and mild solutions coincide. Hence, one might argue that the asymptotic properties (i.e., exponential stability or instability) of solutions to (IVP1) and (IVP2) are the same. For this, we note here that for mild solutions it does not make sense to talk about pointwise properties since mild solutions are in a certain $L_{2}$-space, only. We shall however introduce the correct concepts anticipating this problem. It will turn out that the exponential weight in the $L_{2}$-spaces considered in this exposition can be used to define asymptotically stable parts.

We assume throughout this section that $\mathcal{M}$ is a regular linear operator pencil associated with $\left(M_{0}, M_{1}\right)$ and that the range of $M_{0}$ is a closed subspace of $H$. Moreover, we consider the linear pencil $\mathcal{N}$ associated with $\left(M_{0},-M_{1}\right)$. Since $\mathcal{M}$ is regular, we have by Proposition 3.2 that $\sigma(\mathcal{M})$ is compact and that $\left\|\mathcal{M}(z)^{-1}\right\|$ is uniformly bounded outside a ball $B(0, \rho) \subseteq \mathbb{C}$ for some $\rho>0$ large enough. Since

$$
\mathcal{N}(z)=z M_{0}-M_{1}=-\left(-z M_{0}+M_{1}\right)=-\mathcal{M}(-z), \quad(z \in \mathbb{C})
$$

we infer that also $\mathcal{N}$ is regular and $\left\|\mathcal{N}(z)^{-1}\right\|$ is uniformly bounded outside the same ball $B(0, \rho)$. We first show that there is a strong connection between the solutions of the initial value problems induced by $\mathcal{M}$ and by $\mathcal{N}$. 
We recall from Theorem 5.4 that for each $u_{0} \in H$ the mild solution $u$ of (IVP1) satisfies

$$
u=\mathcal{L}_{\rho}^{*}\left(t \mapsto \frac{1}{\sqrt{2 \pi}}(\mathcal{M}(\mathrm{i} t+\rho))^{-1} M_{0} u_{0}\right) .
$$

Moreover, we have that $\mathbb{R}_{\geq 0} \ni t \mapsto\left(M_{0} u\right)(t)$ is a continuous function. Hence, pointevaluation of $M_{0} u$ is well-defined. This will be used in the next statement.

Proposition 7.1. Let $\mathcal{N}$ be the linear pencil associated with $\left(M_{0},-M_{1}\right)$ and $\rho>0$ large enough, such that $\sup _{z \in \mathbb{C} \backslash B(0, \rho)}\left\|\mathcal{M}(z)^{-1}\right\|<\infty$. Moreover, let $u_{0} \in H$ and let $u \in L_{2, \rho}(\mathbb{R} ; H)$ be given by

$$
u:=\mathcal{L}_{\rho}^{*}\left(t \mapsto \frac{1}{\sqrt{2 \pi}}(\mathcal{M}(\mathrm{i} t+\rho))^{-1} M_{0} u_{0}\right) .
$$

Let $T>0$ and define

$$
w:=\mathcal{L}_{\rho}^{*}\left(t \mapsto \frac{1}{\sqrt{2 \pi}}(\mathcal{N}(\mathrm{i} t+\rho))^{-1}\left(M_{0} u\right)(T)\right) .
$$

Then $w=u(T-\cdot)$ on $[0, T]$.

Proof. We consider the following function

$$
v(t):=\chi_{\mathbb{R}_{\geq 0}}(t+T)(w(t+T)-u(-t)) \quad(t \in \mathbb{R}) .
$$

We note that $v \in L_{2, \rho}(\mathbb{R} ; H)$ since $u, w \in L_{2, \rho}(\mathbb{R} ; H)$ and spt $u \subseteq \mathbb{R}_{\geq 0}$ by Theorem 5.4 . We claim that

$$
\left(\mathcal{L}_{\rho} v\right)(t)=(\mathcal{N}(\mathrm{i} t+\rho))^{-1} M_{0} u_{0} .
$$

If the latter is true, the assertion follows, since then spt $v \subseteq \mathbb{R}_{\geq 0}$ by Theorem 5.4 and thus,

$$
w(t)-u(T-t)=v(t-T)=0 \quad(t \in[0, T] \text { a.e. }) .
$$

To verify (11), we compute using that $\operatorname{spt} u$, spt $w \subseteq \mathbb{R}_{\geq 0}$,

$$
\left(\mathcal{L}_{\rho} v\right)(t)=\mathrm{e}^{(\mathrm{i} t+\rho) T}\left(\mathcal{L}_{\rho} w(t)\right)-\frac{1}{\sqrt{2 \pi}} \int_{-T}^{0} \mathrm{e}^{-(\mathrm{i} t+\rho) s} u(-s) \mathrm{d} s \quad(t \in \mathbb{R})
$$

and thus,

$$
\mathcal{N}(\mathrm{i} t+\rho)\left(\mathcal{L}_{\rho} v\right)(t)=\mathrm{e}^{(\mathrm{i} t+\rho) T} \frac{1}{\sqrt{2 \pi}}\left(M_{0} u\right)(T)-\frac{1}{\sqrt{2 \pi}} \int_{-T}^{0} \mathrm{e}^{-(\mathrm{i} t+\rho) s} \mathcal{N}(\mathrm{i} t+\rho) u(-s) \mathrm{d} s \quad(t \in \mathbb{R}) .
$$

Since $u$ satisfies (2) by Theorem 5.4, we may compute

$$
\int_{-T}^{0} \mathrm{e}^{-(\mathrm{i} t+\rho) s} \mathcal{N}(\mathrm{i} t+\rho) u(-s) \mathrm{d} s
$$




$$
\begin{aligned}
& =\int_{-T}^{0} \mathrm{e}^{-(\mathrm{i} t+\rho) s}\left((\mathrm{i} t+\rho) M_{0} u(-s)-M_{1} u(-s)\right) \mathrm{d} s \\
& =\int_{-T}^{0} \mathrm{e}^{-(\mathrm{i} t+\rho) s}\left((\mathrm{i} t+\rho) M_{0} u_{0}-(\mathrm{i} t+\rho) \int_{0}^{-s} M_{1} u(r) \mathrm{d} r-M_{1} u(-s)\right) \mathrm{d} s .
\end{aligned}
$$

Since

$$
\begin{aligned}
\int_{-T}^{0}-\mathrm{e}^{-(\mathrm{i} t+\rho) s}(\mathrm{i} t+\rho) \int_{0}^{-s} M_{1} u(r) \mathrm{d} r \mathrm{~d} s & =\int_{0}^{T} M_{1} u(r) \int_{-T}^{-r}-\mathrm{e}^{-(\mathrm{i} t+\rho) s}(\mathrm{i} t+\rho) \mathrm{d} s \mathrm{~d} r \\
& =\int_{0}^{T} M_{1} u(r)\left(\mathrm{e}^{(\mathrm{i} t+\rho) r}-\mathrm{e}^{(\mathrm{i} t+\rho) T}\right) \mathrm{d} r \\
& =\int_{-T}^{0} M_{1} u(-s)\left(\mathrm{e}^{-(\mathrm{i} t+\rho) s}-\mathrm{e}^{(\mathrm{i} t+\rho) T}\right) \mathrm{d} s
\end{aligned}
$$

we infer that

$$
\begin{aligned}
& \int_{-T}^{0} \mathrm{e}^{-(\mathrm{i} t+\rho) s} \mathcal{N}(\mathrm{i} t+\rho) u(-s) \mathrm{d} s \\
& =-\left(1-\mathrm{e}^{(\mathrm{i} t+\rho) T}\right) M_{0} u_{0}-\int_{-T}^{0} \mathrm{e}^{-(\mathrm{i} t+\rho) s} M_{1} u(-s) \mathrm{d} s+\int_{-T}^{0} M_{1} u(-s)\left(\mathrm{e}^{-(\mathrm{i} t+\rho) s}-\mathrm{e}^{(\mathrm{i} t+\rho) T}\right) \mathrm{d} s \\
& =\mathrm{e}^{(\mathrm{i} t+\rho) T}\left(M_{0} u_{0}-\int_{-T}^{0} M_{1} u(-s) \mathrm{d} s\right)-M_{0} u_{0} \\
& =\mathrm{e}^{(\mathrm{i} t+\rho) T}\left(M_{0} u\right)(T)-M_{0} u_{0},
\end{aligned}
$$

where we have again used Theorem 5.4, Summarising, we obtain

$$
\begin{aligned}
\mathcal{N}(\mathrm{i} t+\rho)\left(\mathcal{L}_{\rho} v\right)(t) & =\mathrm{e}^{(\mathrm{i} t+\rho) T} \frac{1}{\sqrt{2 \pi}}\left(M_{0} u\right)(T)-\frac{1}{\sqrt{2 \pi}} \int_{-T}^{0} \mathrm{e}^{-(\mathrm{i} t+\rho) s} \mathcal{N}(\mathrm{i} t+\rho) u(-s) \mathrm{d} s \\
& =\frac{1}{\sqrt{2 \pi}} M_{0} u_{0},
\end{aligned}
$$

which proves (11).

We introduce the following two solution operators: Let $\mathcal{N}$ the linear pencil associated with $\left(M_{0},-M_{1}\right)$ and let $\rho>0$ such that $\sup _{z \in \mathbb{C} \backslash B(0, \rho)}\left\|\mathcal{M}(z)^{-1}\right\|<\infty$. Then we define

$$
S(\mathcal{M}): H \rightarrow L_{2, \rho}(\mathbb{R} ; H)
$$




$$
u_{0} \mapsto \mathcal{L}_{\rho}^{*}\left(t \mapsto \frac{1}{\sqrt{2 \pi}}(\mathcal{M}(\mathrm{i} t+\rho))^{-1} M_{0} u_{0}\right)
$$

and

$$
\begin{aligned}
S(\mathcal{N}): H & \rightarrow L_{2, \rho}(\mathbb{R} ; H) \\
w_{0} & \mapsto \mathcal{L}_{\rho}^{*}\left(t \mapsto \frac{1}{\sqrt{2 \pi}}(\mathcal{N}(\mathrm{i} t+\rho))^{-1} M_{0} w_{0}\right) .
\end{aligned}
$$

Using this notation, we realise that Proposition 7.1 states that for all $u_{0} \in H$ and $T>0$

$$
S(\mathcal{N})\left(\left(S(\mathcal{M}) u_{0}\right)(T)\right)=\left(S(\mathcal{M}) u_{0}\right)(T-\cdot)
$$

on $[0, T]$.

Definition 7.2. Let $S \subseteq$ IV be a closed subspace. Then we call $S$ invariant under $\mathcal{M}$, if for all $u_{0} \in S$, we have that $u(t) \in S$ for all $t \in \mathbb{R}_{>0}$, where $u$ is the (strong) solution of (IVP1).

Remark 7.3. Let $\rho>0$ be such that $\sup _{z \in \mathbb{C} \backslash B(0, \rho)}\left\|\mathcal{M}(z)^{-1}\right\|<\infty$. By Proposition 5.1 , we note that the invariance of the closed subspace $S \subseteq$ IV is equivalent to the condition

$$
S(\mathcal{M})[S] \subseteq L_{2, \mu}(\mathbb{R} ; S)
$$

for all $\mu \geq \rho$, which in turn is equivalent to

$$
\left((\mathcal{M}(\mathrm{i} t+\mu))^{-1} M_{0}\right)[S] \subseteq S
$$

for every $t \in \mathbb{R}, \mu \geq \rho$. By the identity theorem, the latter is equivalent to

$$
\left((\mathcal{M}(z))^{-1} M_{0}\right)[S] \subseteq S
$$

for all $z \in \mathbb{C} \backslash B(0, \rho)$.

Definition 7.4. We say that $\mathcal{M}$ admits a strong exponential dichotomy, if there exist closed subspaces $S, T \subseteq$ IV with the following properties: $S+T=\mathrm{IV}, S$ and $T$ are invariant under $\mathcal{M}$ and there is $\rho>0, C \geq 0$ such that for all $t \geq 0$

$$
\begin{cases}|u(t)|_{H} \leq C \mathrm{e}^{-\rho t}\left|u_{0}\right|_{H} & u_{0} \in S, \\ |u(t)|_{H} \geq C \mathrm{e}^{\rho t}\left|u_{0}\right|_{H} & u_{0} \in T,\end{cases}
$$

where $u:=S(\mathcal{M}) u_{0}$. Moreover, we say that $\mathcal{M}$ is strongly exponentially stable, if it admits a strong exponential dichotomy with $S=\mathrm{IV}$ and $T=\{0\}$.

We now reformulate the exponential growth on the subspace $T$ as an exponential decay for the pencil $\mathcal{N}$. We note that the IV-spaces for $\mathcal{M}$ and $\mathcal{N}$ coincide, which is a consequence of IV $=M_{1}^{-1}\left[R\left(M_{0}\right)\right]=-M_{1}^{-1}\left[R\left(M_{0}\right)\right]$, by the linearity of $M_{1}$ and $M_{0}$. 
Lemma 7.5. Let $T \subseteq \mathrm{IV}$ be a closed subspace and invariant under $\mathcal{M}, \rho>0$. Then the following statements are equivalent:

(i) There exists $C>0$ such that

$$
\left|\left(S(\mathcal{M}) u_{0}\right)(t)\right|_{H} \geq C \mathrm{e}^{\rho t}\left|u_{0}\right|_{H}
$$

for all $t \geq 0, u_{0} \in T$.

(ii) There exists $C>0$ such that

$$
\left|\left(S(\mathcal{N}) w_{0}\right)(t)\right|_{H} \leq C \mathrm{e}^{-\rho t}\left|w_{0}\right|_{H}
$$

for all $t \geq 0, w_{0} \in T$.

Proof. (i) $\Rightarrow$ (ii): Let $w_{0} \in T$ and set $w:=S(\mathcal{N}) w_{0}$. We first prove that $w(t) \in T$ for each $t \geq 0$. Indeed, since

$$
\left((\mathcal{M}(z))^{-1} M_{0}\right)[T] \subseteq T,
$$

we infer that also

$$
\left((\mathcal{N}(z))^{-1} M_{0}\right)[T]=\left(-(\mathcal{M}(-z))^{-1} M_{0}\right)[T] \subseteq T
$$

for all $z \in \mathbb{C} \backslash B\left(0, \rho^{\prime}\right)$ for $\rho^{\prime}$ large enough. Hence, $T$ is invariant under $\mathcal{N}$ and thus $w(t) \in T$ for each $t \geq 0$. We fix $t>0$ and set $u:=S(\mathcal{M})(w(t))$. Since $w(t) \in T$ we obtain

$$
|u(s)|_{H} \geq C \mathrm{e}^{\rho s}|w(t)|_{H}
$$

for each $s \geq 0$. Using now Proposition 7.1 (and interchanging the roles of $\mathcal{M}$ and $\mathcal{N}$ ), we derive

$$
u=w(t-\cdot)
$$

on $[0, t]$ and hence, in particular

$$
u(t)=w(0)=w_{0} .
$$

Thus, (12) gives

$$
|w(t)|_{H} \leq C^{-1} \mathrm{e}^{-\rho t}\left|w_{0}\right|_{H}
$$

which shows the claim.

(ii) $\Rightarrow$ (i): The claim can be shown by following the same argumentation as above.

Lemma 7.6. Let $S \subseteq \mathrm{IV}$ be a closed subspace. Then the following conditions are equivalent

(i) $S$ is invariant under $\mathcal{M}$ and there exists $C>0, \rho>0$ such that

$$
\left|\left(S(\mathcal{M}) u_{0}\right)(t)\right|_{H} \leq C \mathrm{e}^{-\rho t}\left|u_{0}\right|_{H}
$$

for all $u_{0} \in S, t \geq 0$. 
(ii) There exists $\rho^{\prime}>0$ such that

$$
S(\mathcal{M}) u_{0} \in L_{2,-\rho^{\prime}}(\mathbb{R} ; S)
$$

for all $u_{0} \in S$.

Proof. (i) $\Rightarrow$ (ii): This is clear for each $0<\rho^{\prime}<\rho$.

(ii) $\Rightarrow(\mathrm{i})$ : Let $u_{0} \in S$. As $u:=S(\mathcal{M}) u_{0}$ is continuous, we infer $\left(S(\mathcal{M}) u_{0}\right)(t) \in S$ for every $t \geq 0$. Let now

$$
\varphi(t):= \begin{cases}1-t & \text { if } 0 \leq t \leq 1, \\ 0 & \text { else. }\end{cases}
$$

Then we compute for each $t \geq 1$ using the representation of $u(t)$ from Corollary 5.2

$$
\begin{aligned}
|u(t)| & =\left|\int_{0}^{t}\left(u^{\prime}(s)-\varphi^{\prime}(s) u_{0}\right) \mathrm{d} s\right| \\
& \leq\left(\int_{0}^{t}\left|u^{\prime}(s)-\varphi^{\prime}(s) u_{0}\right|^{2} \mathrm{e}^{2 \rho^{\prime} s} \mathrm{~d} s\right)^{\frac{1}{2}} \frac{1}{\sqrt{2 \rho^{\prime}}} \mathrm{e}^{-\rho^{\prime} t} \\
& \leq \frac{1}{\sqrt{2 \rho^{\prime}}} \mathrm{e}^{-\rho^{\prime} t}\left(\left|\left(\iota_{R\left(M_{0}\right)}^{*} M_{0} \iota_{\mathrm{IV}}\right)^{-1}\left(\iota_{R\left(M_{0}\right)}^{*} M_{1} \iota_{\mathrm{IV}}\right) u\right|_{L_{2,-\rho^{\prime}}}+\left|u_{0}\right|_{H} \mathrm{e}^{2 \rho^{\prime}}\right) \\
& \leq C \mathrm{e}^{-\rho^{\prime} t}\left(|u|_{L_{2,-\rho^{\prime}}}+\left|u_{0}\right|_{H}\right) .
\end{aligned}
$$

Since $S(\mathcal{M}): S \rightarrow L_{2,-\rho^{\prime}}(\mathbb{R} ; S)$ is closed, we infer its boundedness by the closed graph theorem. Hence, for some $C^{\prime} \geq 0$

$$
|u(t)| \leq C^{\prime} \mathrm{e}^{-\rho^{\prime} t}\left|u_{0}\right|_{H} \quad(t \geq 1) .
$$

By Corollary 5.2 again, we deduce for some $C^{\prime \prime} \geq 0$

$$
|u(t)| \leq C^{\prime \prime} \mathrm{e}^{-\rho^{\prime} t}\left|u_{0}\right|_{H} \quad(t \geq 0)
$$

Thus, the assertion follows with $\rho=\rho^{\prime}$.

With the latter two lemmas at hand, we immediately obtain the following proposition.

Proposition 7.7. $\mathcal{M}$ admits a strong exponential dichotomy if and only if there are two closed subspaces $S, T \subseteq \mathrm{IV}$ with $S \dot{+} T=\mathrm{IV}$ and $\rho>0$ such that

$$
\begin{cases}S(\mathcal{M}) u_{0} \in L_{2,-}(\mathbb{R} ; S), & u_{0} \in S, \\ S(\mathcal{N}) u_{0} \in L_{2,-}(\mathbb{R} ; T) & u_{0} \in T\end{cases}
$$

where $\mathcal{N}$ denotes the pencil associated with $\left(M_{0},-M_{1}\right)$. 
Since this characterisation of a strong exponential dichotomy does not require any continuity conditions on the solutions, we can use this notion to define exponential dichotomies for our second initial value problem (IVP2). For this, observe that by Theorem 5.4, we obtain that the unique solution $u$ solving (IVP2) for $u_{0} \in N\left(M_{0}\right)$ is the zero function. Thus, for the description of asymptotic behaviour, we can dispense with the space $N\left(M_{0}\right)$.

Definition 7.8. We say that $\mathcal{M}$ admits a mild exponential dichotomy if there exist closed subspaces $S, T \subseteq N\left(M_{0}\right)^{\perp}$ satisfying the following properties: $S \dot{+} T=N\left(M_{0}\right)^{\perp}$ and there exists $\rho>0$ such that

$$
\begin{cases}\iota_{N\left(M_{0}\right)^{\perp}}^{*} S(\mathcal{M}) u_{0} \in L_{2,-\rho}(\mathbb{R} ; S), & u_{0} \in S, \\ \iota_{N\left(M_{0}\right)^{\perp}}^{*} S(\mathcal{N}) u_{0} \in L_{2,-\rho}(\mathbb{R} ; T), & u_{0} \in T .\end{cases}
$$

We say that $\mathcal{M}$ is mildly exponentially stable, if it admits a mild exponential dichotomy with $S=N\left(M_{0}\right)^{\perp}$ and $T=\{0\}$.

The main goal is now to provide a characterisation for exponential dichotomy in terms of the spectrum $\sigma_{\mathrm{IV}}(\mathcal{M})=\sigma(\mathcal{M})$. As a prerequisite, we start to study the simple case $M_{0}=1$; moreover, we shall characterise mild and strong exponential stability. Note that we recover the stability theorem in [2, Theorem 3-1.1] for the finite-dimensional case.

Lemma 7.9. Let $M \in L(H), \rho_{1} \in \mathbb{R}$, and let $\widetilde{\mathcal{M}}$ be the pencil associated with $(1, M)$. Then the following conditions are equivalent:

(i) For all $v \in H$ we have that $S(\widetilde{\mathcal{M}}) v \in \bigcap_{\rho>\rho_{1}} L_{2, \rho}(\mathbb{R} ; H)$.

(ii) $\sigma(-M) \subseteq \mathbb{C}_{\operatorname{Re} \leq \rho_{1}}$.

Proof. We show that (i) implies (ii), first. Since $M$ is a bounded operator, its spectrum is compact. Let $\rho_{2}>\rho_{1}$ with $\sigma(-M) \subseteq \mathbb{C}_{\operatorname{Re}<\rho_{2}}$. Assume by contradiction that $\sigma(-M) \cap$ $\mathbb{C}_{\operatorname{Re}>\rho_{1}} \neq \emptyset$. The mapping

$$
\varrho(-M) \cap \mathbb{C}_{\mathrm{Re}>\rho_{1}} \ni z \mapsto(z+M)^{-1}
$$

is holomorphic. By assumption we find a convergent sequence $\left(z_{n}\right)_{n}$ in $\mathbb{C}_{\mathrm{Re}>\rho_{1}}$ such that, for every $n \in \mathbb{N}, z_{n}$ lies in the component of $\mathbb{C}_{\operatorname{Re}>\rho_{2}}$ in $\varrho(-M)$ and

$$
\left\|\left(z_{n}+M\right)^{-1}\right\| \rightarrow \infty \quad(n \rightarrow \infty) .
$$

By the uniform boundedness principle, there exists $v \in H$ such that

$$
\left|\left(z_{n}+M\right)^{-1} v\right|_{H} \rightarrow \infty \quad(n \rightarrow \infty)
$$

Since $S(\widetilde{\mathcal{M}}) v \in \bigcap_{\rho>\rho_{1}} L_{2, \rho}(\mathbb{R} ; H)$ and $\operatorname{spt} S(\widetilde{\mathcal{M}}) v \subseteq \mathbb{R}_{\geq 0}$ by Theorem 5.4, we deduce that the mapping

$$
f: \mathbb{C}_{\operatorname{Re}>\rho_{1}} \ni z \mapsto\left(\mathcal{L}_{\operatorname{Re} z} S(\widetilde{\mathcal{M}}) v\right)(\operatorname{Im} z)=\frac{1}{\sqrt{2 \pi}} \int_{0}^{\infty} \mathrm{e}^{-z t}(S(\widetilde{\mathcal{M}}) v)(t) \mathrm{d} t
$$


is holomorphic. Moreover, by (i), we get

$$
f(z)=\frac{1}{\sqrt{2 \pi}}(z+M)^{-1} v
$$

for all $z \in \mathbb{C}_{\operatorname{Re}>\rho_{2}}$. By the identity theorem, we infer

$$
f\left(z_{n}\right)=\frac{1}{\sqrt{2 \pi}}\left(z_{n}+M\right)^{-1} v
$$

for all $n \in \mathbb{N}$. Since, $f$ is bounded on compact subsets of $\mathbb{C}_{\operatorname{Re}>\rho_{1}}$, we obtain

$$
\infty>\sup _{n}\left|f\left(z_{n}\right)\right|_{H}=\sup _{n}\left|\frac{1}{\sqrt{2 \pi}}\left(z_{n}+M\right)^{-1} v\right|_{H}=\infty,
$$

a contradiction.

Next, we prove that (ii) implies (i). Let $v \in H$ and set $u:=S(\widetilde{\mathcal{M}}) v$. By Theorem 5.4 we have

$$
\left(\mathcal{L}_{\rho} u\right)(t)=\frac{1}{\sqrt{2 \pi}}((\mathrm{i} t+\rho)+M)^{-1} v \quad(t \in \mathbb{R})
$$

for each $\rho>\max \left\{s_{0}(\widetilde{\mathcal{M}}), 0\right\}$. By assumption, the mapping

$$
\begin{aligned}
f: \mathbb{C}_{\operatorname{Re}>\rho_{1}} & \rightarrow H \\
z & \mapsto \frac{1}{\sqrt{2 \pi}}(z+M)^{-1} v
\end{aligned}
$$

is analytic. For $\left.\rho^{\prime} \in\right] \rho_{1}, \rho$, we in particular deduce that $f:\left\{z \in \mathbb{C} ; \rho^{\prime} \leq \operatorname{Re} z \leq \rho\right\} \rightarrow H$ is continuous and analytic in the interior. Since for $|z|>2\|M\|$ we have

$$
|z f(z)| \leq \frac{1}{\sqrt{2 \pi}}|v|_{H}\left\|\left(1+z^{-1} M\right)^{-1}\right\| \leq \sqrt{\frac{2}{\pi}}|v|_{H}
$$

by the Neumann series, we infer

$$
\sup _{\rho^{\prime} \leq \operatorname{Re} z \leq \rho}|z f(z)|<\infty
$$

and thus,

$$
u=\mathcal{L}_{\rho^{\prime}}^{*}\left(f\left(\mathrm{i} \cdot+\rho^{\prime}\right)\right) \in L_{2, \rho^{\prime}}(\mathbb{R} ; H)
$$

according to Lemma 5.5 .

Due to Corollary 4.5 the next result is a direct consequence of Lemma 7.9.

Theorem 7.10. $\mathcal{M}$ admits a strong exponential dichotomy, if, and only if,

$$
\sigma_{\mathrm{IV}}(\mathcal{M})=\sigma\left(-\left(\iota_{R\left(M_{0}\right)}^{*} M_{0} \iota_{\mathrm{IV}}\right)^{-1}\left(\iota_{R\left(M_{0}\right)}^{*} M_{1} \iota_{\mathrm{IV}}\right)\right) \cap i \mathbb{R}=\emptyset .
$$

The pencil $\mathcal{M}$ is exponentially stable, if, and only if,

$$
\sigma_{\mathrm{IV}}(\mathcal{M}) \subseteq \mathbb{C}_{\mathrm{Re}<0}
$$


Proof. If $\mathcal{M}$ admits a strong exponential dichotomy, then $\left(\iota_{R\left(M_{0}\right)}^{*} M_{0} \iota_{\mathrm{IV}}\right)^{-1}\left(\iota_{R\left(M_{0}\right)}^{*} M_{1} \iota_{\mathrm{IV}}\right)$ leaves the spaces $S$ and $T$ invariant. Hence,

$$
\begin{aligned}
& \sigma\left(-\left(\iota_{R\left(M_{0}\right)}^{*} M_{0} \iota_{\mathrm{IV}}\right)^{-1}\left(\iota_{R\left(M_{0}\right)}^{*} M_{1} \iota_{\mathrm{IV}}\right)\right) \\
& =\sigma\left(-\iota_{S}^{*}\left(\iota_{R\left(M_{0}\right)}^{*} M_{0} \iota_{\mathrm{IV}}\right)^{-1}\left(\iota_{R\left(M_{0}\right)}^{*} M_{1} \iota_{\mathrm{IV}}\right) \iota_{S}\right) \\
& \quad \cup \sigma\left(-\iota_{T}^{*}\left(\iota_{R\left(M_{0}\right)}^{*} M_{0} \iota_{\mathrm{IV}}\right)^{-1}\left(\iota_{R\left(M_{0}\right)}^{*} M_{1} \iota_{\mathrm{IV}}\right) \iota_{T}\right) \\
& \subseteq \mathbb{C}_{\operatorname{Re}<0} \cup \mathbb{C}_{\operatorname{Re}>0}
\end{aligned}
$$

by Lemma 7.6 and Lemma 7.9, On the other hand, if $\sigma_{\mathrm{IV}}(\mathcal{M}) \cap \mathrm{i} \mathbb{R}=\emptyset$, then we can choose $S:=P[\mathrm{IV}]$ and $T:=(1-P)[\mathrm{IV}]$, where $P$ denotes the Dunford projection on $\sigma_{\mathrm{IV}}(\mathcal{M}) \cap \mathbb{C}_{\mathrm{Re}<0}$. Indeed, we then obtain that $S$ and $T$ are left invariant and that

$$
\begin{aligned}
& \sigma\left(-\iota_{S}^{*}\left(\iota_{R\left(M_{0}\right)}^{*} M_{0} \iota_{\mathrm{IV}}\right)^{-1}\left(\iota_{R\left(M_{0}\right)}^{*} M_{1} \iota_{\mathrm{IV}}\right) \iota_{S}\right) \subseteq \mathbb{C}_{\mathrm{Re}<0} \\
& \sigma\left(-\iota_{T}^{*}\left(\iota_{R\left(M_{0}\right)}^{*} M_{0} \iota_{\mathrm{IV}}\right)^{-1}\left(\iota_{R\left(M_{0}\right)}^{*} M_{1} \iota_{\mathrm{IV}}\right) \iota_{T}\right) \subseteq \mathbb{C}_{\mathrm{Re}>0},
\end{aligned}
$$

which again yields the assertion by Lemma 7.6 and Lemma 7.9 .

Next, we shall address mild exponential dichotomy and stability. For this, we discuss a preliminary observation first.

Lemma 7.11. Adopt the notation from Proposition 3.2. Then for $z \in \mathbb{C}_{\operatorname{Re}>\rho}$, where $\rho>\max \left\{s_{0}(\mathcal{M}), 0\right\}$, we have

$$
\iota_{N\left(M_{0}\right)^{\perp}}^{*} \mathcal{M}(z)^{-1} M_{0}=(z-A)^{-1} \iota_{N\left(M_{0}\right)^{\perp}}^{*},
$$

where $A:=-\widetilde{M}_{0}^{-1} \widetilde{M}_{1}$ with $\widetilde{M}_{0}, \widetilde{M}_{1}$ iven as in Proposition 3.2. In particular,

$$
\iota_{N\left(M_{0}\right)^{\perp}}^{*} S(\mathcal{M}) u_{0}=S(\widetilde{\mathcal{M}}) \iota_{N\left(M_{0}\right)^{\perp}}^{*} u_{0}
$$

for each $u_{0} \in H$, where $\widetilde{\mathcal{M}}$ denotes the pencil associated with $(1,-A)$.

Proof. By Proposition 3.2 we compute,

$$
\begin{aligned}
& V_{0} U_{0} \mathcal{M}(z)^{-1} M_{0}
\end{aligned}
$$

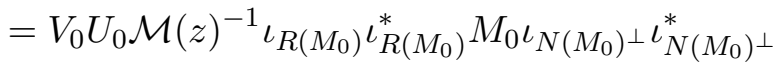

$$
\begin{aligned}
& =\left(\begin{array}{cc}
\left(z \widetilde{M}_{0}+\widetilde{M}_{1}\right)^{-1} & 0 \\
0 & \left(\iota_{R\left(M_{0}\right)^{\perp}}^{*} M_{1} \iota_{N\left(M_{0}\right)}\right)^{-1}
\end{array}\right) V_{1}^{-1} U_{1} \iota_{R\left(M_{0}\right)} \iota_{R\left(M_{0}\right)}^{*} M_{0} \iota_{N\left(M_{0}\right)^{\perp} \iota_{N\left(M_{0}\right)^{\perp}}^{*}}
\end{aligned}
$$




$$
\begin{aligned}
= & \left(\begin{array}{cc}
\left(z \widetilde{M}_{0}+\widetilde{M}_{1}\right)^{-1} & 0 \\
0 & \left(\iota_{R\left(M_{0}\right)^{\perp}}^{*} M_{1} \iota_{N\left(M_{0}\right)}\right)^{-1}
\end{array}\right) V_{1}^{-1}\left(\begin{array}{c}
\iota_{R\left(M_{0}\right)}^{*} \\
\iota_{R\left(M_{0}\right)^{\perp}}^{*}
\end{array}\right) \iota_{R\left(M_{0}\right)} \widetilde{M}_{0} \iota_{N\left(M_{0}\right)^{\perp}}^{*} \\
= & \left(\begin{array}{cc}
\left(z \widetilde{M}_{0}+\widetilde{M}_{1}\right)^{-1} & 0 \\
0 & \left(\iota_{R\left(M_{0}\right)^{\perp}}^{*} M_{1} \iota_{N\left(M_{0}\right)}\right)^{-1}
\end{array}\right) \\
& \times\left(\begin{array}{cc}
1 & -\iota_{R\left(M_{0}\right)^{*}}^{*} M_{1} \iota_{N\left(M_{0}\right)}\left(\iota_{R\left(M_{0}\right)^{\perp}}^{*} M_{1} \iota_{N\left(M_{0}\right)}\right)^{-1} \\
0 & 1
\end{array}\right)\left(\begin{array}{c}
\widetilde{M}_{0} \iota_{N\left(M_{0}\right)^{\perp}}^{*} \\
0
\end{array}\right) \\
= & \left(\begin{array}{c}
\left(z \widetilde{M}_{0}+\widetilde{M}_{1}\right)^{-1} \\
0
\end{array} \quad \begin{array}{c}
\widetilde{M}_{0} \iota_{N\left(M_{0}\right)^{\perp}}^{*} \\
0
\end{array}\right) \\
= & \left(\begin{array}{c}
\left(z \widetilde{M}_{0}+\widetilde{M}_{1}\right)^{-1} \widetilde{M}_{0\left(M_{0} \iota^{\perp}\right.}^{*} M_{N\left(M_{0}\right)^{\perp}}
\end{array}\right) \\
0 & \left.\left(z+\widetilde{M}_{0\left(M_{0}\right)}^{-1} \widetilde{M}_{1}\right)^{-1} \iota_{N\left(M_{0}\right)^{\perp}}^{*}\right)=\left(\begin{array}{c}
(z-A)^{-1} \iota_{N\left(M_{0}\right)^{\perp}}^{*} \\
0
\end{array}\right) .
\end{aligned}
$$

Hence,

$$
\begin{aligned}
\iota_{N\left(M_{0}\right)^{\perp}}^{*} \mathcal{M}(z)^{-1} M_{0} & =\iota_{N\left(M_{0}\right)^{\perp}}^{*} U_{0}^{*} V_{0}^{-1}\left(\begin{array}{c}
(z-A)^{-1} \iota_{N\left(M_{0}\right)^{\perp}}^{*} \\
0
\end{array}\right) \\
& =\iota_{N\left(M_{0}\right)^{\perp}}^{*}\left(\iota_{N\left(M_{0}\right)^{\perp}} \iota_{N\left(M_{0}\right)}\right)\left(\begin{array}{c}
(z-A)^{-1} \iota_{N\left(M_{0}\right)^{\perp}}^{*} \\
0
\end{array}\right) \\
& =(z-A)^{-1} \iota_{N\left(M_{0}\right)^{\perp}}^{*} .
\end{aligned}
$$

Theorem 7.12. $\mathcal{M}$ admits a mild exponential dichotomy, if, and only if,

$$
\sigma(\mathcal{M}) \cap i \mathbb{R}=\emptyset .
$$

$\mathcal{M}$ is exponentially stable, if, and only if,

$$
\sigma(\mathcal{M}) \subseteq \mathbb{C}_{\operatorname{Re}<0}
$$

Proof. By Lemma 7.11, $\mathcal{M}$ admits a mild exponential dichotomy, if $\widetilde{\mathcal{M}}$ admits a mild exponential dichotomy with the same subspaces $S, T$, where $\widetilde{\mathcal{M}}$ denotes the pencil associated with $(1,-A)$. The latter is equivalent to $\sigma(A) \cap i \mathbb{R}=\emptyset$. Indeed, if $\sigma(A) \cap i \mathbb{R}=\emptyset$, we set $S:=P\left[N\left(M_{0}\right)^{\perp}\right]$ and $T:=(1-P)\left[N\left(M_{0}\right)^{\perp}\right]$, where $P$ denotes the Dunford projection on $\sigma(A) \cap \mathbb{C}_{\operatorname{Re}<0}$. The assertion then follows by Lemma 7.6. If on the other hand, $\widetilde{\mathcal{M}}$ admits an exponential dichotomy with invariant subspaces $S, T \subseteq N\left(M_{0}\right)^{\perp}$, we obtain

$$
\sigma(A)=\sigma\left(\iota_{S}^{*} A \iota_{S}\right) \cup \sigma\left(\iota_{T}^{*} A \iota_{T}\right) \subseteq \mathbb{C}_{\mathrm{Re}<0} \cup \mathbb{C}_{\mathrm{Re}>0}
$$

again by Lemma 7.6. Since $\sigma(\mathcal{M})=\sigma(A)$ by Proposition 3.2, the assertion follows. 
From Section 6, Theorem 7.10 and Theorem 7.12 we derive the following statement.

Corollary 7.13. $\mathcal{M}$ admits a strong exponential dichotomy if and only if it admits a mild exponential dichotomy. Moreover, $\mathcal{M}$ is strongly exponentially stable if and only if it is mildly exponentially stable.

\section{References}

[1] T. Berger, A. Ilchmann, and S. Trenn. The quasi-Weierstraß form for regular matrix pencils. Linear Algebra Appl., 436(10):4052-4069, 2012.

[2] L. Dai. Singular Control Systems. Springer-Verlag New York, Inc., Secaucus, NJ, USA, 1989.

[3] E. Griepentrog and R. März. Differential-algebraic equations and their numerical treatment, volume 88 of Teubner-Texte zur Mathematik /Teubner Texts in Mathematics]. BSB B. G. Teubner Verlagsgesellschaft, Leipzig, 1986. With German, French and Russian summaries.

[4] A. Kalauch, R. Picard, S. Siegmund, S. Trostorff, and M. M. Waurick. A Hilbert Space Perspective on Ordinary Differential Equations with Memory Term. Journal of Dynamics and Differential Equations, 26(2):369-399, 2014.

[5] P. Kunkel and V. Mehrmann. Differential-algebraic equations. Analysis and numerical solution. Zürich: European Mathematical Society Publishing House, 2006.

[6] A. S. Markus. Introduction to the spectral theory of polynomial operator pencils, volume 71 of Translations of Mathematical Monographs. American Mathematical Society, Providence, RI, 1988. Translated from the Russian by H. H. McFaden, Translation edited by Ben Silver, With an appendix by M. V. Keldysh.

[7] D. Morgenstern. Beiträge zur nichtlinearen Funktionalanalysis. PhD thesis, TU Berlin, 1952.

[8] R. E. A. C. Paley and N. Wiener. Fourier transforms in the complex domain, volume 19 of American Mathematical Society Colloquium Publications. American Mathematical Society, Providence, RI, 1987. Reprint of the 1934 original.

[9] R. Picard. Hilbert space approach to some classical transforms, volume 196 of Pitman Research Notes in Mathematics Series. Longman Scientific \& Technical, Harlow; copublished in the United States with John Wiley \& Sons, Inc., New York, 1989.

[10] R. Picard. A structural observation for linear material laws in classical mathematical physics. Mathematical Methods in the Applied Sciences, 32:1768-1803, 2009.

[11] R. Picard, S. Trostorff, and M. Waurick. A Functional Analytic Perspective to Delay Differential Equations. Oper. Matrices, 8(1):217-236, 2014. 
[12] R. Picard, S. Trostorff, and M. Waurick. On Evolutionary Equations with Material Laws Containing Fractional Integrals. Math. Meth. Appl. Sci., 38(15):3141-3154, 2015.

[13] T. Reis. Systems Theoretic Aspects of PDAEs and Applications to Electrical Circuits. $\mathrm{PhD}$ thesis, Universität Kaiserslautern, 2006.

[14] W. Rudin. Real and complex analysis. Mathematics series. McGraw-Hill, 1987.

[15] T. Stykel. On criteria for asymptotic stability of differential-algebraic equations. ZAMM Z. Angew. Math. Mech., 82(3):147-158, 2002.

[16] S. Trostorff. Exponential stability for linear evolutionary equations. Asymptot. Anal., 85(3-4):179-197, 2013.

[17] S. Trostorff. Exponential stability for second order evolutionary problems. J. Math. Anal. Appl., 429(2):1007-1032, 2015.

[18] S. Trostorff. Exponential Stability and Initial Value Problems for Evolutionary Equations. Habilitation thesis, TU Dresden, 2017. arXiv:1707.00429.

[19] S. Trostorff and M. Waurick. On Higher Index DAEs in Infinite Dimensions. Technical report, TU Dresden, University of Strathclyde, 2017 arXiv:1710.08750. 